\title{
An approximate $\kappa$ state solutions of the Dirac equation for the generalized Morse potential under spin and pseudospin symmetry
}

\author{
Sameer M. Ikhdair ${ }^{1, *}$ \\ ${ }^{1}$ Physics Department, Near East University, Nicosia, N. Cyprus, Turkey
}

(Dated: August 29, 2018)

\begin{abstract}
By using an improved approximation scheme to deal with the centrifugal (pseudo-centrifugal) term, we solve the Dirac equation for the generalized Morse potential with arbitrary spin-orbit quantum number $\kappa$. In the presence of spin and pseudospin symmetry, the analytic bound state energy eigenvalues and the associated upper- and lower-spinor components of two Dirac particles are found by using the basic concepts of the Nikiforov-Uvarov method. We study the special cases when $\kappa= \pm 1(l=\widetilde{l}=0, s$-wave), the non-relativistic limit and the limit when $\alpha$ becomes zero (Kratzer potential model). The present solutions are compared with those obtained by other methods.

Keywords: Dirac equation, spin symmetry, pseudospin symmetry, generalized Morse potential, approximation schemes; Nikiforov-Uvarov method

PACS numbers: 03.65.Pm; 03.65.Ge; 03.65.-w; 03.65.Fd; 02.30.Gp
\end{abstract}

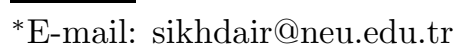




\section{INTRODUCTION}

The simplest modified Morse potential model suggested by Deng and Fan [1] and related to the Manning-Rosen potential [2] (also called Eckart potential by some authors [3]) is anharmonic potential defined by

$$
V(r)=D\left(1-\frac{b}{e^{\alpha r}-1}\right)^{2}, b=e^{\alpha r_{e}}-1,
$$

where $r \in(0, \infty)$, and the three positive parameters $D, r_{e}$ and $\alpha$ denote the dissociation energy, the equilibrium inter-nuclear distance and the range of the potential well, respectively. The above potential is used to describe diatomic molecular energy spectra and electromagnetic transitions and is the true internuclear potential in diatomic molecules with the same behaviour for $r \rightarrow 0$ [4]. The above potential was called a generalized Morse potential (GMP) model and illustrated in Figure 1 for various values of potential parameters. As stated in Ref. [4], the Morse potential and the GMP are very close to each other for large values of $r_{e}$ $(\alpha=1)$ in the regions $r \sim r_{e}$ and $r>r_{e}$ but are very different at $r \sim 0$. Further, if the two potentials are deep $(D \gg 1)$, they could be well approximated by a harmonic oscillator in the region $r \sim r_{e}$ (see Fig.1 in [4]). To describe the vibrational spectra of diatomic molecules the potential curve $V(r)$ is approximated by a sum of three Morse functions [5]. The approach has the advantage of being more flexible than the simple Morse potential while preserving the correct asymptotical $V(\infty)=$ const [5]. The potential model (1) is a special case of the five-parameter exponential-type potential model $[6,7]$. The exact solvability of the $s$-wave ( $l=0$ case) bound state energy eigenvalues and eigenfunctions of the GMP is due to the fact that it belongs to the class of the Eckart potential, a member of the hypergeometric Natanzon potentials which can be solved algebraically by means of $S O(2,2)$ symmetry algebra [4] and $S O(2,1)$ algebra [8]. The X-H stretching motion in small molecules has been treated by the potential model (1) [9]. Moreover, the approximated $l$-wave bound state solutions [10] of the Schrödinger equation has been solved using the conventional approximation scheme suggested by Greene and Aldrich [11] to deal with the centrifugal barrier term $l(l+1) / r^{2}$, singularity at $r=0$. The exact analytic expressions for matrix elements of positive integral powers of the coordinate [12] and the quasi-one-dimensional system of DNA [13] have also been studied.

To study the relativistic effects and corrections of the molecular Morse potential [4], the 
Dirac equation has been solved for attractive scalar $S(r)$ and repulsive vector $V(r)$ Morse potentials under pseudospin symmetry in the nuclear theory using the Pekeris approximation [14]. Recently, the approximate bound state solutions of the pseudospin and spin symmetric Dirac equation with the GMP has been calculated using an improved approximation scheme to deal with the centrifugal (pseudo-centrifugal) term and by employing the basic concept of the supersymmetric shape invariance formalism (cf. [15] and the references therein).

Many authors have investigated approximately the solution of the Dirac equation with a few potential models such as the Hulthén potential [16], the Hulthen potential including Coulomb-like tensor potential [17], the generalized Woods-Saxon potential [18,19], the Eckart potential [20], the Morse potential [14,21], the Pöschl-Teller potential [22], the ManningRosen potential [2,23], the hyperbolic potential [24], the Rosen-Morse potential [25], the pseudoharmonic potential [26], and the Kratzer potential connected with an angle-dependent potential [27], etc within the framework of various methods.

The diatomic molecular model consisting of nuclei having masses $m_{1}$ and $m_{2}$, the reduced mass is defined $\mu=m_{1} m_{2} /\left(m_{1}+m_{2}\right)$ can be included to the spin symmetry and the pseudospin symmetry concepts [28]. Ginocchio [29-31] showed that the spin symmetry occurs when the difference potential between the vector potential $V(r)$ and scalar potential $S(r)$ is a constant (i.e., $\Delta(r)=V(r)-S(r)=C_{s}=$ constant) and the pseudospin symmetry occurs when the sum potential of the vector potential $V(r)$ and scalar potential $S(r)$ is a constant (i.e., $\Sigma(r)=V(r)-S(r)=C_{p s}=$ constant). The spin symmetry concept [32] is particularly relevant for mesons [33]. The pseudospin symmetry concept [34,35] in nuclear physics refers to the quasi-degeneracy of single-nucleon doublets and can be characterized with the nonrelativistic quantum numbers $(n, l, j=l+1 / 2)$ and $(n-1, l+2, j=l+3 / 2)$, where $n, l$ and $j$ are the single-nucleon radial, orbital and total angular momentum quantum numbers, respectively. Alhaidari et al. [36] investigated in detail the physical interpretation on the three-dimensional Dirac equation in the cases of spin symmetry limitation $\Delta(r)=0$ and pseudospin symmetry limitation $\Sigma(r)=0$. In real nuclei, $\Sigma(r) \neq$ constant and pseudospin symmetry is only an approximation. The quality of the pseudospin symmetry approximation depends on the competition between the pseudo-centrifugal potential and the pseudospin orbital potential [37].

Recently, in the framework of the spin symmetry $S(r) \sim V(r)$ and pseudospin symmetry $S(r) \sim-V(r)$, the bound state energy eigenvalues and associated upper- and lower-spinor 
wave functions are investigated by means of the Nikiforov-Uvarov (NU) method [38]. We have approximately solved the Dirac equation for the Rosen-Morse potential [25] with spin and pseudospin symmetry for any $\kappa$ state and found the eigenvalue equation and corresponding two-component spinors within the framework of an approximation to the term proportional to $1 / r^{2}$. We have also solved the $(3+1)$ dimensional Dirac equation for a particle trapped in the spherically symmetric generalized WS potential under the conditions of exact spin and pseudospin symmetry combined with approximation for the spin-orbit centrifugal (pseudo-centrifugal) term, and calculated the two-component spinor wave functions and the energy eigenvalues for any arbitrary spin-orbit $\kappa$ bound states [18].

In principle, the solution of the Schrödinger equation for the GMP model can be used to describe the motion of the nucleons in the mean field produced by the interactions between nuclei. However, the Dirac equation successfully merges quantum mechanics with special relativity and is considered to be the natural transition to quantum field theory. It provides a natural description of the electron spin, predicts the existence of antimatter and is able to reproduce accurately the spectrum of the hydrogen atom. It also predicts some peculiar phenomena such as Klein's paradox and unexpected quivering motion of free relativistic quantum particle which are key examples for understanding relativistic quantum effects, but are difficult to observe in real particles. In order to to understand the origin of spin and pseudospin symmetry, we need to take into consideration the motion of the nucleons in a relativistic mean field and consider the Dirac equation [31]. We attempt to study the approximate solution of the Dirac equation for the GMP with non-zero spin-orbit quantum numbers $\kappa$ by employing an improved approximation scheme (see e.g., $[17,39,40]$ and the references therein) to deal with the spin-orbit centrifugal, $\kappa(\kappa+1) / r^{2}$ (pseudo-centrifugal, $\left.\kappa(\kappa-1) / r^{2}\right)$ barrier term. The inter-relation between the GMP with the Oscillator and Morse potentials investigated in Ref. [4] and the recent relativistic treatments of the Morse and Oscillator potentials in $[14,26]$ are some motivations for the present study. The simple transformation of the Dirac equation into the Schrödinger-like equation and the success in studying the approximated bound state solutions of the Dirac equation with various potential models $[14,17,18,25,26]$ also make the solution possible.

We tend to show that the new scheme of parametric generalization of the NU method [41] given in Appendix $\mathrm{A}$ is a powerful tool for solving a second order differential equation by turning it into a hypergeometric type equation [38]. The advantage of employing the 
NU method, in the present work, is that it can be used to find the bound state energy spectra and the corresponding spinor wave functions under the condition of spin symmetry and pseudospin symmetry concept for any $\kappa$ state in a very simple way.

This paper is organized as follows. In section 2, we investigate the bound state energy equation and the corresponding two-component spinor wave functions under the condition of spin symmetry and pseudospin symmetry concept for the GMP model by employing a parametric generalization of the NU method. In section 3, we study some special cases like the $s(\widetilde{s}$ )-wave cases $\kappa= \pm 1(l=\widetilde{l}=0)$, the non-relativistic limit and the $\alpha \rightarrow 0$ (Kratzer potential). In section 4 , we present some numerical results to the non-relativistic and relativistic numerical energy levels for GMP and Kratzer models. The relevant conclusions are given in section 5 .

\section{DIRAC BOUND STATE SOLUTIONS}

The Dirac equation for fermionic massive spin-1/2 particles moving in an attractive scalar potential $S(r)$ and a repulsive vector potential $V(r)$ is given by [42]

$$
\left[c \boldsymbol{\alpha} \cdot \mathbf{p}+\boldsymbol{\beta}\left(M c^{2}+S(r)\right)+V(r)-E\right] \psi_{n \kappa}(\mathbf{r})=0, \psi_{n \kappa}(\mathbf{r})=\psi(r, \theta, \phi),
$$

where $E$ is the relativistic energy of the system, $m$ is the mass of a particle, $\mathbf{p}=-i \hbar \boldsymbol{\nabla}$ is the momentum operator, and $\boldsymbol{\alpha}$ and $\boldsymbol{\beta}$ are $4 \times 4$ Dirac matrices, i.e.,

$$
\boldsymbol{\alpha}=\left(\begin{array}{cc}
0 & \boldsymbol{\sigma}_{i} \\
\boldsymbol{\sigma}_{i} & 0
\end{array}\right), \boldsymbol{\beta}=\left(\begin{array}{cc}
\mathbf{I} & 0 \\
0 & -\mathbf{I}
\end{array}\right), \sigma_{1}=\left(\begin{array}{ll}
0 & 1 \\
1 & 0
\end{array}\right), \sigma_{2}=\left(\begin{array}{cc}
0 & -i \\
i & 0
\end{array}\right), \sigma_{3}=\left(\begin{array}{cc}
1 & 0 \\
0 & -1
\end{array}\right),
$$

where $\mathbf{I}$ denotes the $2 \times 2$ identity matrix and $\boldsymbol{\sigma}_{i}$ are the three-vector Pauli spin matrices. For a spherical symmetrical nuclei, the total angular momentum operator of the nuclei $\mathbf{J}$ and spin-orbit matrix operator $\mathbf{K}=-\boldsymbol{\beta}(\boldsymbol{\sigma} \cdot \mathbf{L}+\mathbf{I})$ commute with the Dirac Hamiltonian, where $\mathbf{L}$ is the orbital angular momentum operator. The spinor wave functions can be classified according to the radial quantum number $n$ and the spin-orbit quantum number $\kappa$ and can be written using the Pauli-Dirac representation in the following forms:

$$
\psi_{n \kappa}(\mathbf{r})=\left(\begin{array}{c}
f_{n \kappa}(\mathbf{r}) \\
g_{n \kappa}(\mathbf{r})
\end{array}\right)=\frac{1}{r}\left(\begin{array}{c}
F_{n \kappa}(r) Y_{j m \kappa}^{l}(\widehat{r}) \\
i G_{n \kappa}(r) Y_{j m(-\kappa)}^{\tilde{l}}(\widehat{r})
\end{array}\right),
$$

where the upper- and lower-spinor components $F_{n \kappa}(r)$ and $G_{n \kappa}(r)$ are the real square-integral radial wave functions, $Y_{j m \kappa}^{l}(\widehat{r})$ and $Y_{j m(-\kappa)}^{\widetilde{l}}(\widehat{r})$ are the spin spherical harmonic functions 
coupled to the total angular momentum $j$ and it's projection $m$ on the $z$ axis and $\kappa(\kappa+1)=$ $l(l+1)$ and $\kappa(\kappa-1)=\widetilde{l}(\widetilde{l}+1)$. The quantum number $\kappa$ is related to the quantum numbers for spin symmetry $l$ and pseudospin symmetry $\widetilde{l}$ as

$$
\kappa=\left\{\begin{array}{ccl}
-(l+1)=-\left(j+\frac{1}{2}\right), & \left(s_{1 / 2}, p_{3 / 2}, \text { etc. }\right), & j=l+\frac{1}{2}, \quad \text { aligned } \operatorname{spin}(\kappa<0), \\
+l=+\left(j+\frac{1}{2}\right), & \left(p_{1 / 2}, d_{3 / 2}, \text { etc. }\right), & j=l-\frac{1}{2}, \quad \text { unaligned } \operatorname{spin}(\kappa>0),
\end{array}\right.
$$

and the quasi-degenerate doublet structure can be expressed in terms of a pseudospin angular momentum $\widetilde{s}=1 / 2$ and pseudo-orbital angular momentum $\widetilde{l}$ which is defined as

$$
\kappa=\left\{\begin{array}{cl}
-\widetilde{l}=-\left(j+\frac{1}{2}\right), & \left(s_{1 / 2}, \quad p_{3 / 2}, \quad \text { etc. }\right), \quad j=\tilde{l}-1 / 2, \quad \text { aligned } \operatorname{spin}(\kappa<0), \\
+(\tilde{l}+1)=+\left(j+\frac{1}{2}\right), & \left(d_{3 / 2}, \quad f_{5 / 2}, \quad \text { etc. }\right), \quad j=\tilde{l}+1 / 2, \quad \text { unaligned } \operatorname{spin}(\kappa>0),
\end{array}\right.
$$

where $\kappa= \pm 1, \pm 2, \cdots$. For example, $\left(1 s_{1 / 2}, 0 d_{3 / 2}\right)$ and $\left(2 \mathrm{p}_{3 / 2}, 1 f_{5 / 2}\right)$ can be considered as pseudospin doublets.

Upon direct substitution of Eq. (4) into Eq. (2), we can obtain two radial coupled Dirac equations for the two spinor components as follows:

$$
\begin{aligned}
& \left(\frac{d}{d r}+\frac{\kappa}{r}\right) F_{n \kappa}(r)=\left[M c^{2}+E_{n \kappa}-\Delta(r)\right] G_{n \kappa}(r), \\
& \left(\frac{d}{d r}-\frac{\kappa}{r}\right) G_{n \kappa}(r)=\left[M c^{2}-E_{n \kappa}+\Sigma(r)\right] F_{n \kappa}(r),
\end{aligned}
$$

where $\Delta(r)=V(r)-S(r)$ and $\Sigma(r)=V(r)+S(r)$ are the difference and sum potentials, respectively.

Under the spin symmetry ( i.e., $\Delta(r)=C_{s}=$ constant), one can eliminate $G_{n \kappa}(r)$ in Eq. (7a), with the aid of Eq. (7b), to obtain a second-order differential equation for the upper-spinor component as follows:

$$
\begin{gathered}
{\left[-\frac{d^{2}}{d r^{2}}+\frac{\kappa(\kappa+1)}{r^{2}}+\frac{1}{\hbar^{2} c^{2}}\left(M c^{2}+E_{n \kappa}-C_{s}\right) \Sigma(r)\right] F_{n \kappa}(r)} \\
=\frac{1}{\hbar^{2} c^{2}}\left(E_{n \kappa}^{2}-M^{2} c^{4}+C_{s}\left(M c^{2}-E_{n \kappa}\right)\right) F_{n \kappa}(r),
\end{gathered}
$$

and the lower-spinor component is obtained from Eq. (7a) as

$$
G_{n \kappa}(r)=\frac{1}{M c^{2}+E_{n \kappa}-C_{s}}\left(\frac{d}{d r}+\frac{\kappa}{r}\right) F_{n \kappa}(r)
$$

where $E_{n \kappa} \neq-M c^{2}$, only real positive energy states exist when $C_{s}=0$ (exact spin symmetry). On the other hand, under the pseudospin symmetry ( i.e., $\Sigma(r)=C_{p s}=$ constant), 
one can eliminate $F_{n \kappa}(r)$ in Eq. (7b), with the aid of Eq. (7a), to obtain a second-order differential equation for the lower-spinor component as follows:

$$
\begin{gathered}
{\left[-\frac{d^{2}}{d r^{2}}+\frac{\kappa(\kappa-1)}{r^{2}}-\frac{1}{\hbar^{2} c^{2}}\left(M c^{2}-E_{n \kappa}+C_{p s}\right) \Delta(r)\right] G_{n \kappa}(r)} \\
=\frac{1}{\hbar^{2} c^{2}}\left(E_{n \kappa}^{2}-M^{2} c^{4}-C_{p s}\left(M c^{2}+E_{n \kappa}\right)\right) G_{n \kappa}(r),
\end{gathered}
$$

and the upper-spinor component $F_{n \kappa}(r)$ is obtained from Eq. (7b) as

$$
F_{n \kappa}(r)=\frac{1}{M c^{2}-E_{n \kappa}+C_{p s}}\left(\frac{d}{d r}-\frac{\kappa}{r}\right) G_{n \kappa}(r)
$$

where $E_{n \kappa} \neq M c^{2}$, only real negative energy states exist when $C_{p s}=0$ (exact pseudospin symmetry). It is worthy to note that the reality and finiteness of our solutions demand that the upper and lower radial components should satisfy the essential boundary conditions: $F_{n \kappa}(0)=G_{n \kappa}(0)=0$ and $F_{n \kappa}(\infty)=G_{n \kappa}(\infty)=0$.

\section{A. Spin symmetry solutions of the GMP model}

At first, we investigate the spin symmetry by taking the $\Sigma(r)=2 V(r) \rightarrow V_{G M P}(r)$ as mentioned in Ref. [43] enables one to reduce the resulting relativistic solutions into their non-relativistic limit under appropriate transformations. From Eq. (8), we can see that the energy eigenvalues, $E_{n \kappa}$, depend only on $n$ and $l$, i.e., $E_{n \kappa}=E(n, l(l+1))$. For $l \neq 0$, the states with $j=l \pm 1 / 2$ are degenerate. This is a $S U(2)$ spin symmetry. Following Refs. [22-25], we impose the GMP $[1,4]$ as the $\Sigma(r)$, i.e.,

$$
\Sigma(r)=D\left(1-\frac{b}{e^{\alpha r}-1}\right)^{2},
$$

leads us to obtain a Schrödinger-like equation in the form:

$$
\begin{gathered}
{\left[\frac{d^{2}}{d r^{2}}-\frac{\kappa(\kappa+1)}{r^{2}}-\alpha^{2} \nu_{1}^{2}\left(1-\frac{b e^{-\alpha r}}{1-e^{-\alpha r}}\right)^{2}+\alpha^{2} \omega_{1}^{2}\right] F_{n \kappa}(r)=0} \\
\nu_{1}^{2}=\frac{1}{\alpha^{2} \hbar^{2} c^{2}}\left(M c^{2}+E_{n \kappa}-C_{s}\right) D, \quad \omega_{1}^{2}=\frac{1}{\alpha^{2} \hbar^{2} c^{2}}\left[E_{n \kappa}^{2}-M^{2} c^{4}+C_{s}\left(M c^{2}-E_{n \kappa}\right)\right],
\end{gathered}
$$

where $\kappa(\kappa+1)=l(l+1), \kappa=l$ for $\kappa<0$ and $\kappa=-(l+1)$ for $\kappa>0$. The exact solution of the above equation is possible only for the $s$-wave case $(\kappa=-1)$ due to the centrifugal term $\kappa(\kappa+1) / r^{2}$. However, if $\kappa$ is not too large, the case of the vibrations of small amplitude 
about the minimum, we attempt to use the following improved new approximation scheme to deal with the centrifugal (pseudo centrifugal) term, near the minimum point $r=r_{e}$ (i.e., $x=0)$, (cf. Refs. [17,40,44-48]):

$\frac{1}{r^{2}} \approx \alpha^{2}\left[d_{0}+\frac{e^{-\alpha r}}{\left(1-e^{-\alpha r}\right)^{2}}\right]=\alpha^{2}\left[d_{0}+\frac{1}{(\alpha r)^{2}}-\frac{1}{12}+\frac{(\alpha r)^{2}}{240}-\frac{(\alpha r)^{4}}{6048}+\frac{(\alpha r)^{6}}{172800}+O\left((\alpha r)^{8}\right)\right]$.

When $\alpha r \ll 1$, the value of the dimensionless constant $d_{0}=1 / 12$ has simply determined by the above series expansion and $\alpha$ takes the unit of reciprocal of $r$. The present approximation was shown to be more powerful than the usual approximation [40]. Obviously, the above approximation to the centrifugal (pseudo-centrifugal) term turns to $1 / r^{2}$ when the parameter $\alpha$ goes to zero as

$$
\lim _{\alpha \rightarrow 0}\left[\alpha^{2}\left(d_{0}+\frac{1}{e^{\alpha r}-1}+\frac{1}{\left(e^{\alpha r}-1\right)^{2}}\right)\right]=\frac{1}{r^{2}},
$$

which shows that the usual approximation is the limit of our approximation (cf. e.g., $[17,40]$ and the references therein).

We introduce the following new dimensionless parameter, $\mathrm{z}(r)=e^{-\alpha r} \in[0,1]$, which maintains the finiteness of the transformed wave functions on the boundary conditions. Thus, substituting Eq. (15) into Eq. (13), we obtain the following Schrödinger-like equation satisfying $F_{n \kappa}(r)$,

$$
\begin{gathered}
\left(\frac{d^{2}}{d z^{2}}+\frac{(1-z)}{z(1-z)} \frac{d}{d z}\right) F_{n \kappa}(z) \\
+\frac{1}{z^{2}(1-z)^{2}}\left\{-\left[(2+b) b \nu_{1}^{2}+\varepsilon_{n \kappa}^{2}\right] z^{2}+\left[2\left(b \nu_{1}^{2}+\varepsilon_{n \kappa}^{2}\right)-\kappa(\kappa+1)\right] z-\varepsilon_{n \kappa}^{2}\right\} F_{n \kappa}(z)=0,
\end{gathered}
$$

where

$$
\varepsilon_{n \kappa}^{2}=\nu_{1}^{2}-\omega_{1}^{2}+\kappa(\kappa+1) d_{0},
$$

and $F_{n \kappa}(r)=F_{n \kappa}(z)$. In order to clarify the parametric generalization of the NU method [25], let us take the following general form of a Schrödinger-like equation written for any potential,

$$
\frac{d^{2} \psi_{n}}{d z^{2}}+\frac{\widetilde{\tau}(z)}{\sigma(z)} \frac{d \psi_{n}}{d z}+\frac{\widetilde{\sigma}(z)}{\sigma^{2}(z)} \psi_{n}(z)=0,
$$

satisfying the wave functions

$$
\psi_{n}(z)=\phi(z) y_{n}(z)
$$

where

$$
\widetilde{\tau}(z)=c_{1}-c_{2} z
$$


and

$$
\sigma(z)=z\left(1-c_{3} z\right) \text { and } \tilde{\sigma}(z)=-A z^{2}+B z-C
$$

are two polynomials at most of first- and second-degree, respectively. Furthermore, when Eq. (17) is compared with its counterpart Eq. (19), we can obtain the specific values for the constants $c_{i}(i=1,2,3)$ along with $A, B$ and $C$. Now, following the NU method [38] and making the substitution of Eqs. (21) and (22), we can obtain general forms for the polynomials $\pi(z)$ and $\tau(z)$, the root of the parameter $k$, the eigenvalues equation and the wave functions $\phi(z)$ and $y_{n}(z)$ expressed in terms of the constants $c_{i}(i=4,5, \cdots, 13)$ as shown in Appendix A (cf. Refs. [18,21,25,40]). Therefore, the task of computing the energy eigenvalues and the corresponding wave functions of Eq. (17) within the framework of the parametric generalization of the NU method is relatively easy and straightforward. It is explained in the following steps. Firstly, we need to find the specific values for the parametric constants $c_{i}(i=4,5, \cdots, 13)$ by means of the relation A1 of Appendix A. The values of all these constants $c_{i}(i=1,2, \cdots, 13)$ together with $A, B$ and $C$ are therefore displayed in Table 1 for the GMP model. Secondly, using the relations (A2-A5), the analytic forms of the essential polynomials $\pi(z)$ and $\tau(z)$ along with the root $k$, required by the method [41], can also be found as

$$
\begin{gathered}
\pi(z)=-\frac{z}{2}-\frac{1}{2}\left[\left(\sqrt{(1+2 \kappa)^{2}+4 b^{2} \nu_{1}^{2}}+2 \varepsilon_{n \kappa}\right)-2 \varepsilon_{n \kappa}\right], \\
k=2 b \nu_{1}^{2}-\kappa(\kappa+1)-\varepsilon_{n \kappa} \sqrt{(1+2 \kappa)^{2}+4 b^{2} \nu_{1}^{2}} \\
\tau(z)=1+2 \varepsilon_{n \kappa}-2\left(1+\varepsilon_{n \kappa}+\frac{1}{2} \sqrt{(1+2 \kappa)^{2}+4 b^{2} \nu_{1}^{2}}\right) z,
\end{gathered}
$$

where $\tau^{\prime}(z)<0$ must be satisfied in order to obtain a physical solution according to the NU method [38]. Thirdly, we need to calculate the energy eigenvalues by means of the eigenvalue equation, relation $\mathrm{A} 6$, and obtain

$$
\varepsilon_{n \kappa}=\frac{(2+b) b \nu_{1}^{2}}{2 n+1+\sqrt{(1+2 \kappa)^{2}+4 b^{2} \nu_{1}^{2}}}-\frac{2 n+1+\sqrt{(1+2 \kappa)^{2}+4 b^{2} \nu_{1}^{2}}}{4} .
$$

Finally, with the aid of Eqs. (14) and (18), Eq. (26) can be also reduced to the energy equation for the GMP with the spin symmetry concept for any spin-orbit quantum number $\kappa= \pm 1, \pm 2, \cdots$ values in the Dirac theory,

$$
\left(M c^{2}+E_{n \kappa}-C_{s}\right)\left(M c^{2}+D-E_{n \kappa}\right)+\hbar^{2} c^{2} \alpha^{2} \kappa(\kappa+1) d_{0}
$$




$$
=\hbar^{2} c^{2} \alpha^{2}\left(\frac{(2+b) b \nu_{1}^{2}}{2\left(n+\delta_{1}\right)}-\frac{\left(n+\delta_{1}\right)}{2}\right)^{2}, n=0,1,2, \cdots
$$

where

$$
\delta_{1}=\frac{1}{2}\left(1+\sqrt{(1+2 \kappa)^{2}+4 b^{2} \nu_{1}^{2}}\right) \geq 1 .
$$

In what follows, in order to establish the wave functions $F_{n \kappa}(r)$ of Eq. (8), the relations (A7-A10) are used. Firstly, we find the first part of the wave functions yields

$$
\phi(z)=z^{\varepsilon_{n \kappa}}(1-z)^{\delta_{1}}, \varepsilon_{n \kappa}>0, \delta_{1}>0 .
$$

Secondly, we calculate the weight function as

$$
\rho(z)=z^{2 \varepsilon_{n \kappa}}(1-z)^{2 \delta_{1}-1}
$$

and this, in turn, generates the second part of the wave functions,

$$
y_{n}(z) \sim z^{-2 \varepsilon_{n \kappa}}(1-z)^{-\left(2 \delta_{1}-1\right)} \frac{d^{n}}{d z^{n}}\left[z^{n+2 \varepsilon_{n \kappa}}(1-z)^{n+2 \delta_{1}-1}\right] \approx P_{n}^{\left(2 \varepsilon_{n \kappa}, 2 \delta_{1}-1\right)}(1-2 z),
$$

where $P_{n}^{(a, b)}(1-2 z)$ is the orthogonal Jacobi polynomials [49,50]. Finally, the upper spinor component $F_{n \kappa}(z)$ for arbitrary $\kappa$ can be obtained by means of Eq. (20) as

$$
\begin{gathered}
F_{n \kappa}(r)=\mathcal{N}_{n \kappa} e^{-\varepsilon_{n \kappa} \alpha r}\left(1-e^{-\alpha r}\right)^{\delta_{1}} P_{n}^{\left(2 \varepsilon_{n \kappa}, 2 \delta_{1}-1\right)}\left(1-2 e^{-\alpha r}\right) \\
=\mathcal{N}_{n \kappa} \frac{\Gamma\left(n+2 \varepsilon_{n \kappa}+1\right)}{\Gamma\left(2 \varepsilon_{n \kappa}+1\right) n !} e^{-\varepsilon_{n \kappa} \alpha r}\left(1-e^{-\alpha r}\right)^{\delta_{1}}{ }_{2} F_{1}\left(-n, n+2 \varepsilon_{n \kappa}+2 \delta_{1} ; 1+2 \varepsilon_{n \kappa} ; e^{-\alpha r}\right),
\end{gathered}
$$

where the normalization constants $\mathcal{N}_{n \kappa}$ are calculated in Appendix B.

Let us recall the derivative relation of the hypergeometric function,

$$
\frac{d}{d z}\left[{ }_{2} F_{1}(a ; b ; c ; z)\right]=\left(\frac{a b}{c}\right){ }_{2} F_{1}(a+1 ; b+1 ; c+1 ; z),
$$

that is used to calculate the corresponding lower-component $G_{n \kappa}(r)$ in Eq. (9) as

$$
\begin{gathered}
G_{n \kappa}(r)=\frac{\mathcal{N}_{n \kappa}}{M c^{2}+E_{n \kappa}-C_{s}}\left(\frac{\alpha \delta_{1} e^{-\alpha r}}{1-e^{-\alpha r}}-\alpha \varepsilon_{n \kappa}+\frac{\kappa}{r}\right) F_{n \kappa}(r) \\
+\mathcal{N}_{n \kappa} \frac{n \alpha\left(n+2 \varepsilon_{n \kappa}+2 \delta_{1}\right)}{\left(M c^{2}+E_{n \kappa}-C_{s}\right)\left(1+2 \varepsilon_{n \kappa}\right)}\left(1-e^{-\alpha r}\right)^{\delta_{1}}\left(e^{-\alpha r}\right)^{\varepsilon_{n \kappa}+1} \\
\quad \times{ }_{2} F_{1}\left(1-n ; n+2\left(\varepsilon_{n \kappa}+\delta_{1}\right)+1 ; 2\left(1+\varepsilon_{n \kappa}\right) ; e^{-\alpha r}\right) .
\end{gathered}
$$

We would like to note that the hypergeometric series ${ }_{2} F_{1}\left(1-n ; n+2\left(\varepsilon_{n \kappa}+\delta_{1}\right)+1 ; 2\left(1+\varepsilon_{n \kappa}\right) ; e^{-\alpha r}\right)$ terminates for $n=0$ and thus does not diverge for all values of real parameters $\varepsilon_{n \kappa}$ and $\delta_{1}$. 
In the presence of exact spin symmetry $\left(C_{s}=0\right), E_{n \kappa} \neq-M c^{2}$ (only positive energy states do exist). In the exact spin symmetry $\left(C_{s}=0\right)$ with $(\kappa=1, \kappa=-2)$, the upperand lower- spinor wave functions for the ground $\left(0 p_{1 / 2}, 0 p_{3 / 2}\right)$ and first excited $\left(1 p_{1 / 2}, 1 p_{3 / 2}\right)$ degenerate eigenstates are being illustrated in Figure $2 \mathrm{a}$ and 2b, respectively. A glance at Figure 2 reveals that there is only one curve (dashed line) for the radial wavefunctions of the upper components for both states in the doublet. However, there are two curves (solid lines) for the radial wavefunctions of the lower components. The following values of the parameters $M=1.0 \mathrm{fm}^{-1}, D=15 \mathrm{fm}^{-1}, \alpha=0.1 \mathrm{fm}^{-1}$ and $r_{e}=0.4 \mathrm{fm}, E_{0, \kappa=1}=$ $E_{0, \kappa=-2}=5.5791076 \mathrm{fm}^{-1}$ and $E_{1, \kappa=1}=E_{1, \kappa=-2}=8.1823677 \mathrm{fm}^{-1}$ have been used.

Let us now study the special case when the parameter $\alpha \rightarrow 0$ in Eq. (1). The GMP potential can be easily reduced to the well-known Kratzer molecular potential,

$$
\lim _{\alpha \rightarrow 0} V(r)=D\left(\frac{r-r_{e}}{r}\right)^{2} .
$$

which has been studied extensively by using different methods as the function analysis [51], the NU $[52,53]$ and the exact quantization rule (EQR) [54]. To avoid the repetition, following Appendix A, we write down the essential polynomials:

$$
\begin{gathered}
\pi(r)=\frac{1}{2}\left[1+\gamma-2 \epsilon_{n \kappa} r\right], \\
k=\frac{2 r_{e}}{\hbar^{2} c^{2}}\left(M c^{2}+E_{n \kappa}-C_{s}\right) D-\gamma \epsilon_{n \kappa}, \\
\tau(r)=1+\gamma-2 \epsilon_{n \kappa} r,
\end{gathered}
$$

where

$$
\begin{aligned}
\gamma & =\sqrt{(1+2 \kappa)^{2}+\frac{4 r_{e}^{2}}{\hbar^{2} c^{2}}\left(M c^{2}+E_{n \kappa}-C_{s}\right) D}, \\
\epsilon_{n \kappa} & =\frac{1}{\hbar c} \sqrt{\left(M c^{2}-E_{n \kappa}+D\right)\left(M c^{2}+E_{n \kappa}-C_{s}\right)} .
\end{aligned}
$$

We further obtain the following two expressions which are relevant in the construction of the energy equation (relation A6) [38]

$$
\lambda_{n}=2 n \epsilon_{n \kappa} \text { and } \lambda=\frac{2 r_{e}}{\hbar^{2} c^{2}}\left(M c^{2}+E_{n \kappa}-C_{s}\right) D-(1+\gamma) \epsilon_{n \kappa},
$$

and substituting $\lambda_{n}=\lambda$, we can obtain the following spin symmetric energy equation,

$$
\left(M c^{2}-E_{n \kappa}+D\right)=\frac{q D^{2}\left(M c^{2}+E_{n \kappa}-C_{s}\right)}{\left(N_{n}+\sqrt{N_{\kappa}^{2}+q D\left(M c^{2}+E_{n \kappa}-C_{s}\right)}\right)^{2}},
$$


where $N_{n}=2 n+1, N_{\kappa}=2 \kappa+1$ and $q=\left(2 r_{e} / \hbar c\right)^{2}$. The above equation for energies looks like a quartic equation of the form:

$$
a_{4} E_{n \kappa}^{4}+a_{3} E_{n \kappa}^{3}+a_{2} E_{n \kappa}^{2}+a_{1} E_{n \kappa}+a_{0}=0
$$

with coefficients

$$
\begin{gathered}
a_{4}=q^{2} D^{2} ; a_{3}=2 q D\left\lceil N_{\kappa}^{2}-N_{n}^{2}-q D C_{s}\right\rceil ; \\
a_{2}=\left(N_{n}^{2}-N_{\kappa}^{2}\right)^{2}+2 q D\left(D+M+C_{s}\right)\left(N_{n}^{2}-N_{\kappa}^{2}\right)+4 q D^{2} N_{n}^{2}+q^{2} D^{2}\left(C_{s}^{2}+2 M C_{s}-2 M^{2}\right) \\
a_{1}=2 q^{2} D^{2} M C_{s}\left(M-C_{s}\right)-2(D+M)\left(N_{n}^{2}-N_{\kappa}^{2}\right)^{2} \\
+2 q D\left(M^{2}-2 M C_{s}-D C_{s}\right)\left(N_{n}^{2}-N_{\kappa}^{2}\right)-4 q D^{2}\left(D+C_{s}\right) N_{n}^{2} \\
a_{0}=\left\lceil q D M^{2}-(D+M)\left(N_{n}^{2}-N_{\kappa}^{2}\right)\right\rceil^{2}-4 q D^{2}\left(M-C_{s}\right)(D+M) N_{n}^{2} \\
+q^{2} D^{2} M^{2} C_{s}\left(C_{s}-2 M\right)+2 q D M C_{s}(D+M)\left(N_{n}^{2}-N_{\kappa}^{2}\right) ;
\end{gathered}
$$

where we have set $c=1$. For a given value of $n$ and $\kappa($ or $l$ ), the above quartic equation, Eq. (41b), provides four distinct positive and negative real (real and complex) energy spectra related with $E_{n \kappa}^{+}$or $E_{n \kappa}^{-}$, respectively. One of the distinct solutions is only valid to obtain the positive-energy bound states in the limit of the spin symmetry. Therefore, the procedures for calculating the four distinct energies; namely, $E_{n \kappa}^{(1)}, E_{n \kappa}^{(2)}, E_{n \kappa}^{(3)}$ and $E_{n \kappa}^{(4)}$ are given in Appendix B.

Furthermore, following [18], the normalized upper- and lower-spinor wave functions can be calculated as

$$
F_{n \kappa}(r)=\left(2 \epsilon_{n \kappa}\right)^{K+1} \sqrt{\frac{\epsilon_{n \kappa} n !}{(n+K+1) \Gamma(n+2 K+2)}} r^{K+1} e^{-\epsilon_{n \kappa} r} L_{n}^{(2 K+1)}\left(2 \epsilon_{n \kappa} r\right),
$$

and

$$
\begin{gathered}
G_{n \kappa}(r)=\frac{1}{M c^{2}+E_{n \kappa}-C_{s}}\left(2 \epsilon_{n \kappa}\right)^{K+1} \sqrt{\frac{\epsilon_{n \kappa} n !}{(n+K+1) \Gamma(n+2 K+2)}} \\
\times\left[\left(\frac{(K+1)+\kappa}{r}-\epsilon_{n \kappa}\right) F_{n \kappa}(r)-2 \epsilon_{n \kappa} r^{K+1} e^{-\epsilon_{n \kappa} r} L_{n-1}^{(2 K+2)}\left(2 \epsilon_{n \kappa} r\right)\right] \\
K=\frac{1}{2}(\gamma-1),
\end{gathered}
$$


respectively, where $L_{n}^{(\beta)}(x)$ are associated Laguerre polynomials. The simplest exact spin solution, representing the ground state and first excited state, are

$$
\begin{gathered}
\left\{\begin{array}{c}
\left(M c^{2}+E_{0 \kappa}\right)\left(M c^{2}-E_{0 \kappa}+D\right)=\frac{1}{\hbar^{2} c^{2}}\left(\frac{r_{e}\left(M c^{2}+E_{0 \kappa}\right) D}{1+\sqrt{(1+2 \kappa)^{2}+\frac{4 D r_{e}^{2}}{\hbar^{2} c^{2}}\left(M c^{2}+E_{0 \kappa}\right)}}\right)^{2} \\
F_{0 \kappa}(r)=\left(2 \epsilon_{0 \kappa}\right)^{K_{0}+1} \sqrt{\frac{\epsilon_{0 \kappa}}{\left(K_{0}+1\right) \Gamma\left(2 K_{0}+2\right)}} r^{K_{0}+1} e^{-\epsilon_{n \kappa} r}, \\
G_{0 \kappa}(r)=\frac{1}{M c^{2}+E_{0 \kappa}}\left(2 \epsilon_{0 \kappa}\right)^{K_{0}+1} \sqrt{\frac{\epsilon_{0 \kappa}}{\left(K_{0}+1\right) \Gamma\left(2 K_{0}+2\right)}}\left(\frac{\left(K_{0}+1\right)+\kappa}{r}-\epsilon_{0 \kappa}\right) F_{0 \kappa}(r), \\
\left(M c^{2}+E_{1 \kappa}\right)\left(D+M c^{2}-E_{1 \kappa}\right)=\frac{1}{\hbar^{2} c^{2}}\left(\frac{D r_{e}\left(M c^{2}+E_{1 \kappa}\right)}{3+\sqrt{(1+2 \kappa)^{2}+\frac{4 D r_{e}^{2}}{\hbar^{2} c^{2}}\left(M c^{2}+E_{1 \kappa}\right)}}\right)^{2}, \\
F_{1 \kappa}(r)=\left(2 \epsilon_{1 \kappa}\right)^{K_{1}+1} \sqrt{\frac{\epsilon_{1 \kappa}}{\left(K_{1}+2\right) \Gamma\left(2 K_{1}+3\right)}} r^{K_{1}+1} e^{-\epsilon_{1 \kappa} r}\left(-2 \epsilon_{1 \kappa} r+2 K_{1}+2\right), \\
\frac{1}{M c^{2}+E_{1 \kappa}}\left(2 \epsilon_{1 \kappa}\right)^{K_{1}+1} \sqrt{\frac{\epsilon_{1 \kappa}}{(K+2) \Gamma(2 K+3)}}\left[\left(\frac{\left(K_{1}+1\right)+\kappa}{r}-\epsilon_{1 \kappa}\right) F_{1 \kappa}(r)-2 \epsilon_{1 \kappa} r^{K_{1}+1} e^{-\epsilon_{1 \kappa} r}\right]
\end{array}\right.
\end{gathered}
$$

respectively. Finally, we would like to note that $\epsilon_{0 \kappa}$ and $\epsilon_{1 \kappa}$ can be calculated via Eq. (39) whereas $K_{0}$ and $K_{1}$ via Eq. (43) along with Eq. (38) when $C_{s}=0$.

\section{B. Pseudospin symmetry solutions of the GMP model}

From Eq. (10), we can see that the energy eigenvalues, $E_{n \kappa}$, depend only on $n$ and $\tilde{l}$, i.e., $E_{n \kappa}=E(n, \tilde{l}(\tilde{l}+1))$. For $\widetilde{l} \neq 0$, the states with $j=\widetilde{l} \pm 1 / 2$ are degenerate. This is a $S U(2)$ pseudospin symmetry. Following Refs. [22-25], we impose the GMP [1] as the $\Delta(r)$, i.e.,

$$
\Delta(r)=D\left(1-\frac{b}{e^{\alpha r}-1}\right)^{2}
$$

leads us to obtain a Schrödinger-like equation in the form:

$$
\left[\frac{d^{2}}{d r^{2}}-\frac{\kappa(\kappa-1)}{r^{2}}+\alpha^{2} \nu_{2}^{2}\left(1-\frac{b e^{-\alpha r}}{1-e^{-\alpha r}}\right)^{2}+\alpha^{2} \omega_{2}^{2}\right] G_{n \kappa}(r)=0
$$

where

$$
\nu_{2}^{2}=\frac{1}{\alpha^{2} \hbar^{2} c^{2}}\left(M c^{2}-E_{n \kappa}+C_{p s}\right) D, \quad \omega_{2}^{2}=\frac{1}{\alpha^{2} \hbar^{2} c^{2}}\left[E_{n \kappa}^{2}-M^{2} c^{4}-C_{p s}\left(M c^{2}+E_{n \kappa}\right)\right]
$$

where $\kappa(\kappa-1)=\tilde{l}(\tilde{l}+1)$. We follow the same procedures in the previous subsection to obtain a Dirac equation satisfying $G_{n \kappa}(r)$,

$$
\left(\frac{d^{2}}{d z^{2}}+\frac{(1-z)}{z(1-z)} \frac{d}{d z}\right) G_{n \kappa}(z)
$$




$$
+\frac{1}{z^{2}(1-z)^{2}}\left\{-\left[\widetilde{\varepsilon}_{n \kappa}^{2}-(2+b) b \nu_{2}^{2}\right] z^{2}+\left[2\left(\widetilde{\varepsilon}_{n \kappa}^{2}-b \nu_{2}^{2}\right)-\kappa(\kappa-1)\right] z-\widetilde{\varepsilon}_{n \kappa}^{2}\right\} G_{n \kappa}(z)=0
$$

where

$$
\widetilde{\varepsilon}_{n \kappa}^{2}=-\omega_{2}^{2}-\nu_{2}^{2}+\kappa(\kappa-1) d_{0},
$$

where $G_{n \kappa}(r)=G_{n \kappa}(z)$. To avoid repetition in the solution of Eq. (45), a careful inspection for the relationship between the present set of parameters $\left(\widetilde{\varepsilon}_{n \kappa}, \nu_{2}^{2}\right)$ and the previous one $\left(\varepsilon_{n \kappa}, \nu_{1}^{2}\right)$ tells us that the negative energy solution for pseudospin symmetry, where $S(r) \sim$ $-V(r)$, can be obtained directly from the spin symmetric solution by using the following parameter mapping $[18,55]$ :

$F_{n \kappa}(r) \leftrightarrow G_{n \kappa}(r), \kappa \rightarrow \kappa-1, V(r) \rightarrow-V(r)$ (i.e., $\left.D \rightarrow-D\right), E_{n \kappa} \rightarrow-E_{n \kappa}$ and $C_{s} \rightarrow-C_{p s}$.

Following the previous procedures, the constants in the case of pseudospin symmetry concept are displayed in Table 2. Applying the above transformations to Eqs. (27), (32) and (33) lead to the following pseudospin symmetric energy equation,

$$
\begin{gathered}
\left(E_{n \kappa}-M c^{2}-C_{p s}\right)\left(D-M c^{2}-E_{n \kappa}\right)+\hbar^{2} c^{2} \alpha^{2} \kappa(\kappa-1) d_{0} \\
=\hbar^{2} c^{2} \alpha^{2}\left(\frac{(2+b) b \nu_{2}^{2}}{2\left(n+\delta_{2}\right)}+\frac{\left(n+\delta_{2}\right)}{2}\right)^{2}, n=0,1,2, \cdots,
\end{gathered}
$$

with

$$
\delta_{2}=\frac{1}{2}\left(1+\sqrt{(1-2 \kappa)^{2}-4 b^{2} \nu_{2}^{2}}\right) \geq 1 .
$$

Note that Eq. (50) can be also expressed in the form of quartic equation (cf. Eq. (41b)) since the two parameters $\nu_{2}^{2}$ and $\delta_{2}$ contain the energy eigenvalues $E_{n \kappa}$. The procedures of this analytic solution is so similar to the one presented in Appendix B with the changes $D \rightarrow-D, E_{n \kappa} \rightarrow-E_{n \kappa}$ and $C_{s} \rightarrow-C_{p s}$. Furthermore, the lower-component wave functions:

$$
\begin{gathered}
G_{n \kappa}(r)=\mathcal{N}_{n \kappa} e^{-\widetilde{\varepsilon}_{n \kappa} \alpha r}\left(1-e^{-\alpha r}\right)^{\delta_{2}} P_{n}^{\left(2 \widetilde{\varepsilon}_{n \kappa}, 2 \delta_{2}-1\right)}\left(1-2 e^{-\alpha r}\right) \\
=\mathcal{N}_{n \kappa} \frac{\left(2 \widetilde{\varepsilon}_{n \kappa}+1\right)_{n}}{n !} e^{-\widetilde{\varepsilon}_{n \kappa} \alpha r}\left(1-e^{-\alpha r}\right)_{2}^{\delta_{2}} F_{1}\left(-n, n+2 \widetilde{\varepsilon}_{n \kappa}+2 \delta_{2} ; 1+2 \widetilde{\varepsilon}_{n \kappa} ; e^{-\alpha r}\right),
\end{gathered}
$$

where

$$
\widetilde{\varepsilon}_{n \kappa}=-\left(\frac{(2+b) b \nu_{2}^{2}}{2 n+1+\sqrt{(1-2 \kappa)^{2}-4 b^{2} \nu_{2}^{2}}}+\frac{2 n+1+\sqrt{(1-2 \kappa)^{2}-4 b^{2} \nu_{2}^{2}}}{4}\right)
$$


The upper-component $F_{n \kappa}(r)$ can be calculated from Eq. (11) as follows

$$
\begin{aligned}
& F_{n \kappa}(r)=\frac{\mathcal{N}_{n \kappa}}{M c^{2}-E_{n \kappa}+C_{p s}}\left(\frac{\alpha \delta_{2} e^{-\alpha r}}{1-e^{-\alpha r}}-\alpha \widetilde{\varepsilon}_{n \kappa}-\frac{\kappa}{r}\right) G_{n \kappa}(r) \\
& +\mathcal{N}_{n \kappa} \frac{n \alpha\left(n+2 \widetilde{\varepsilon}_{n \kappa}+2 \delta_{2}\right)}{\left(M c^{2}-E_{n \kappa}+C_{p s}\right)\left(1+2 \widetilde{\varepsilon}_{n \kappa}\right)}\left(1-e^{-\alpha r}\right)^{\delta_{2}}\left(e^{-\alpha r}\right)^{\widetilde{\varepsilon}_{n \kappa}+1} \\
& \quad \times{ }_{2} F_{1}\left(1-n ; n+2\left(\widetilde{\varepsilon}_{n \kappa}+\delta_{2}\right)+1 ; 2\left(1+\widetilde{\varepsilon}_{n \kappa}\right) ; e^{-\alpha r}\right) .
\end{aligned}
$$

Hence, in the exact pseudospin symmetry where $C_{p s}=0, E_{n \kappa} \neq M c^{2}$ (only negative energy states exist).

On the other hand, the pseudospin solutions of the Dirac equation for the Kratzer potential can be obtained from the spin symmetry case by applying transformation map given by Eq. (49) as

$$
\begin{gathered}
\tilde{\gamma}=\sqrt{(1-2 \kappa)^{2}-\frac{4 D r_{e}^{2}}{\hbar^{2} c^{2}}\left(M c^{2}-E_{n \kappa}+C_{p s}\right)}, \\
\tilde{\epsilon}_{n \kappa}=\frac{1}{\hbar c} \sqrt{-D\left(M c^{2}-E_{n \kappa}+C_{p s}\right)-\left(E_{n \kappa}^{2}-M^{2} c^{4}-C_{p s}\left(M c^{2}+E_{n \kappa}\right)\right)} .
\end{gathered}
$$

Therefore, the eigenvalue equation is

$$
\begin{gathered}
E_{n \kappa}^{2}-M^{2} c^{4}-C_{p s}\left(M c^{2}+E_{n \kappa}\right)=-D\left(M c^{2}-E_{n \kappa}+C_{p s}\right) \\
-\frac{1}{\hbar^{2} c^{2}}\left(\frac{D r_{e}\left(M c^{2}-E_{n \kappa}+C_{p s}\right)}{n+K+1}\right)^{2}, \widetilde{K}=\frac{1}{2}(\widetilde{\gamma}-1),
\end{gathered}
$$

and the normalized lower- and upper-spinor wave functions are given by

$$
\begin{aligned}
& G_{n \kappa}(r)=\left(2 \widetilde{\epsilon}_{n \kappa}\right)^{\widetilde{K}+1} \sqrt{\frac{\widetilde{\epsilon}_{n \kappa} n !}{(n+\widetilde{K}+1) \Gamma(n+2 \widetilde{K}+2)}} r^{\widetilde{K}+1} e^{-\widetilde{\epsilon}_{n \kappa} r} L_{n}^{(2 \widetilde{K}+1)}\left(2 \widetilde{\epsilon}_{n \kappa} r\right), \\
& F_{n \kappa}(r)=\frac{1}{M c^{2}-E_{n \kappa}+C_{p s}}\left(2 \widetilde{\epsilon}_{n \kappa}\right)^{\widetilde{K}+1} \sqrt{\frac{\widetilde{\epsilon}_{n \kappa} n !}{(n+\widetilde{K}+1) \Gamma(n+2 \widetilde{K}+2)}} \\
& \quad \times\left[\left(\frac{(\widetilde{K}+1)-\kappa}{r}-\widetilde{\epsilon}_{n \kappa}\right) G_{n \kappa}(r)-2 \widetilde{\epsilon}_{n \kappa} r^{\widetilde{K}+1} e^{-\widetilde{\epsilon}_{n \kappa} r} L_{n-1}^{(2 \widetilde{K}+2)}\left(2 \widetilde{\epsilon}_{n \kappa} r\right)\right] .
\end{aligned}
$$




\section{SOME SPECIAL CASES}

Let us study three special cases. At first, we study the $s(\widetilde{s})$-states $(l=\widetilde{l}=0$, i.e., $\kappa=\mp 1$ ) . It follows that the spin-orbit coupling term $\kappa(\kappa+1) / r^{2}=0$, and also the corresponding approximation to it in Eq. (27). In the presence of the exact spin symmetry limit $\left(C_{s}=0\right)$, the $s$-states $(\kappa=-1)$ energy equation becomes

$$
E_{n,-1}^{2}-M^{2} c^{4}-D\left(M c^{2}+E_{n,-1}\right)=-\hbar^{2} c^{2} \alpha^{2}\left(\frac{\frac{1}{\hbar^{2} c^{2} \alpha^{2}}(2+b)\left(M c^{2}+E_{n,-1}\right) D b}{2(n+\delta)}-\frac{n+\delta}{2}\right)^{2}
$$

where

$$
\delta=\frac{1}{2}\left(1+\sqrt{1+\frac{4}{\hbar^{2} c^{2} \alpha^{2}}\left(M c^{2}+E_{n,-1}\right) D b^{2}}\right) \geq 1 .
$$

The upper- and lower-spinor wave functions are

$$
F_{n,-1}(r)=\mathcal{N}_{n,-1} \frac{(2 \eta+1)_{n}}{n !} e^{-\eta \alpha r}\left(1-e^{-\alpha r}\right)_{2}^{\delta} F_{1}\left(-n, n+2 \eta+2 \delta ; 1+2 \eta ; e^{-\alpha r}\right),
$$

and

$$
\begin{gathered}
G_{n,-1}(r)=\frac{\mathcal{N}_{n,-1}}{M c^{2}+E_{n,-1}}\left(\frac{\alpha \delta e^{-\alpha r}}{1-e^{-\alpha r}}-\alpha \eta-\frac{1}{r}\right) F_{n,-1}(r) \\
+\mathcal{N}_{n,-1} \frac{n \alpha(n+2 \eta+2 \delta)}{\left(M c^{2}+E_{n,-1}\right)(1+2 \eta)}\left(1-e^{-\alpha r}\right)^{\delta}\left(e^{-\alpha r}\right)^{\eta+1} \\
\quad \times{ }_{2} F_{1}\left(1-n ; n+2(\eta+\delta)+1 ; 2(1+\eta) ; e^{-\alpha r}\right),
\end{gathered}
$$

with

$$
\eta=\frac{\frac{1}{\hbar^{2} c^{2} \alpha^{2}}(2+b)\left(M c^{2}+E_{n,-1}\right) D b}{2(n+\delta)}-\frac{n+\delta}{2},
$$

where $\mathcal{N}_{n,-1}$ is calculated in the Appendix C. Overmore, for the $\widetilde{s}$-states $(\kappa=1)$ in the exact pseudospin symmetry $\left(C_{p s}=0\right)$, the energy equation (48) becomes

$$
E_{n 1}^{2}-M^{2} c^{4}+D\left(M c^{2}-E_{n 1}\right)=-\hbar^{2} c^{2} \alpha^{2}\left(\frac{\frac{1}{\hbar^{2} c^{2} \alpha^{2}}(2+b)\left(M c^{2}-E_{n 1}\right) D b}{2\left(n+\delta_{2}\right)}+\frac{\left(n+\delta_{2}\right)}{2}\right)^{2}
$$

with

$$
\delta_{2}=\frac{1}{2}\left(1+\sqrt{1-\frac{4}{\alpha^{2} \hbar^{2} c^{2}}\left(m c^{2}-E_{n 1}\right) D b^{2}}\right), E_{n 1}>m c^{2}, n=0,1,2, \cdots .
$$

The wave functions given by Eqs. (52) and (54) become

$$
G_{n 1}(r)=\mathcal{N}_{n 1} e^{-\eta_{2} \alpha r}\left(1-e^{-\alpha r}\right)^{\delta_{2}} P_{n}^{\left(2 \eta_{2}, 2 \delta_{2}-1\right)}\left(1-2 e^{-\alpha r}\right)
$$




$$
=\mathcal{N}_{n 1} \frac{\left(2 \eta_{2}+1\right)_{n}}{n !} e^{-\eta_{2} \alpha r}\left(1-e^{-\alpha r}\right)^{\delta_{2}}{ }_{2} F_{1}\left(-n, n+2 \eta_{2}+2 \delta_{2} ; 1+2 \eta_{2} ; e^{-\alpha r}\right)
$$

with

$$
\eta_{2}=\frac{(2+b) \xi_{2}}{2 n+1+\sqrt{1+4 b \xi_{2}}}-\frac{2 n+1+\sqrt{1+4 b \xi_{2}}}{4}
$$

and

$$
\begin{aligned}
& F_{n 1}(r)=\frac{\mathcal{N}_{n 1}}{M c^{2}-E_{n 1}}\left(\frac{\alpha \delta_{2} e^{-\alpha r}}{1-e^{-\alpha r}}-\alpha \eta_{2}-\frac{1}{r}\right) G_{n \kappa}(r) \\
& +\mathcal{N}_{n 1} \frac{n \alpha\left(n+2 \eta_{2}+2 \delta_{2}\right)}{\left(M c^{2}-E_{n \kappa}\right)\left(1+2 \eta_{2}\right)}\left(1-e^{-\alpha r}\right)^{\delta_{2}}\left(e^{-\alpha r}\right)^{\eta_{2}+1} \\
& \times{ }_{2} F_{1}\left(1-n ; n+2\left(\eta_{2}+\delta_{2}\right)+1 ; 2\left(1+\eta_{2}\right) ; e^{-\alpha r}\right),
\end{aligned}
$$

where $E_{n 1} \neq M c^{2}$.

Second, we study the nonrelativistic limit. In applying the following appropriate mapping $E_{n \kappa}-M c^{2} \rightarrow E_{n l}$ and $\frac{1}{\hbar^{2} c^{2}}\left(M c^{2}+E_{n \kappa}\right) \rightarrow \frac{2 \mu}{\hbar^{2}}$ to Eqs. (27) and (32), we obtain the energy levels of the Schrödinger equation for any arbitrary orbital quantum number $l$ as

$$
E_{n l}=D+\frac{\hbar^{2}}{2 \mu} l(l+1) \alpha^{2} d_{0}-\frac{\hbar^{2} \alpha^{2}}{2 \mu}\left(\frac{\frac{\mu}{\hbar^{2} \alpha^{2}}(2+b) D b}{n+\widetilde{\delta}}-\frac{n+\widetilde{\delta}}{2}\right)^{2}
$$

and the radial wave functions as

$$
\psi_{n l}(r)=\mathcal{N}_{n l} \frac{(2 \widetilde{\eta}+1)_{n}}{n !} e^{-\widetilde{\eta} \alpha r}\left(1-e^{-\alpha r}\right)^{\widetilde{\delta}}{ }_{2} F_{1}\left(-n, n+2 \widetilde{\eta}+2 \widetilde{\delta} ; 1+2 \widetilde{\eta} ; e^{-\alpha r}\right)
$$

with

$$
\widetilde{\delta}=\frac{1}{2}\left(1+\sqrt{(1+2 l)^{2}+\frac{8 \mu}{\hbar^{2} \alpha^{2}} D b^{2}}\right) \geq 1, \widetilde{\eta}=\frac{\frac{\mu}{\hbar^{2} \alpha^{2}}(2+b) D b}{n+\widetilde{\delta}}-\frac{n+\widetilde{\delta}}{2},
$$

where $\mu=m_{1} m_{2} /\left(m_{1}+m_{2}\right)$ is the reduced mass of the two atoms and $\mathcal{N}_{n l}$ is calculated in the Appendix C.

Third, the case $\alpha \rightarrow 0$, the nonrelativistic limit of the bound state solutions of Eqs. (41) and (42) in exact symmetry limit $\left(C_{s}=0\right)$ can be obtained as

$$
E_{n l}=D--\frac{8 \mu}{\hbar^{2}}\left(\frac{D r_{e}}{1+2 n+\sqrt{(1+2 l)^{2}+\frac{8 \mu}{\hbar^{2}} D r_{e}^{2}}}\right)^{2}
$$

and the normalized wave functions:

$$
\psi_{n l}(r)=\left(2 \widetilde{\epsilon}_{n l}\right)^{L+1} \sqrt{\frac{\widetilde{\epsilon}_{n l} n !}{(n+L+1) \Gamma(n+2 L+2)}} r^{L+1} e^{-\widetilde{\epsilon}_{n l} r} L_{n}^{(2 L+1)}\left(2 \widetilde{\epsilon}_{n l} r\right),
$$


where

$$
\widetilde{\epsilon}_{n l}=\frac{2 \mu D r_{e}}{\hbar^{2}(n+L+1)} \text { and } L=\frac{1}{2}\left(\sqrt{(1+2 l)^{2}+\frac{8 \mu}{\hbar^{2}} D r_{e}^{2}}-1\right)
$$

which are identical to the previous results obtained by the function analysis method [51], the NU method [52,53] and the EQR (cf. Eq. (29) in Ref. [54] obtained in D-dimensions).

\section{NUMERICAL RESULTS}

As in Ref. [56], we calculate the non-relativistic energy levels as function of various values of the parameter that controls the width of the potential well $\alpha=0.05-0.30 \mathrm{fm}^{-1}$ and equilibrium inter-nuclear distance $r_{e}=0.4,0.8 \mathrm{fm}$ for various states with quantum numbers $n$ and $l$. The atomic units $\hbar=M=1$ are used and the dissociation energy is set to $D=15 \mathrm{fm}^{-1}$. In Table 3 , we display our results with those ones calculated by using the conventional approximation scheme suggested by Greene and Aldrich [12] to deal with the centrifugal term $l(l+1) / r^{2}$ together with the values obtained from the numerical integration procedures based on the MATHEMATICA package programmed by Lucha and Schöberl [57]. Obviously, our results are closely approaching the ones obtained in [57] for both short range ( $\operatorname{small} \alpha$ ) as well as for long range (large $\alpha$ ) potential (see e.g., our recent works $[39,58]$ ). This means that our new approximated calculations using the approximation scheme (15) proposed recently by us provides much better approximation to the centrifugal term than that in Ref. [55] even for large $\alpha$ values (see, e.g., [40] and the references therein). At small values of $\alpha$ (Kratzer potential), we have also calculated the energy levels as a function of $r_{e}=0.1-1.5 \mathrm{fm}$ for various quantum numbers $n$ and $l$. Hence, our numerical values of these energy levels are shown in Table 4. Overmore, Table 5 presents some numerical values for the eigeenergies of the Dirac valence states obtained from Eq. (27) with parameters $M=1.0$ $\mathrm{fm}^{-1}, D=15 \mathrm{fm}^{-1}, \alpha=0.1,0.3 \mathrm{fm}^{-1}$ and $r_{e}=0.4,0.8 \mathrm{fm}$ (exact spin symmetry case, i.e., $\left.C_{s}=0 \mathrm{fm}^{-1}\right)$. We noticed that there are only positive energy bound state solutions in the spin symmetry limit. One can also see from Table 5 that there are degeneracies between the eigenstates $\left(n p_{1 / 2}, n p_{3 / 2}\right),\left(n d_{3 / 2}, n d_{5 / 2}\right),\left(n f_{5 / 2}, n f_{7 / 2}\right),\left(n g_{7 / 2}, n g_{9 / 2}\right)$, etc. In fact, each of these eigenstates form a spin doublet. For instance, for any specific value of $n$, where $n=0,1,2, \cdots, n p_{1 / 2}$ with $\kappa=1$ is considered as the partner of $n p_{3 / 2}$ with $\kappa=-2$. Thus, states that have the same radial $n$ and orbital angular momentum $l$ quantum numbers with $j=l+1 / 2$ and $j=l-1 / 2$ are degenerate [59]. 
On the other hand, in Table 6, we present some numerical values for the eigeenergies of the Dirac hole states obtained from Eq. (50) with the previous choice of potential parameters for the case of pseudospin symmetry limit $\left(C_{p s}=0,5.0,-5.0\right.$ and $\left.-10.0 \mathrm{fm}^{-1}\right)$. One can see from Table 6 that there are degeneracies between the eigenstates $\left(n s_{1 / 2},(n-1) d_{3 / 2}\right)$, $\left(n p_{3 / 2},(n-1) f_{5 / 2}\right),\left(n d_{5 / 2},(n-1) g_{7 / 2}\right),\left(n f_{7 / 2},(n-1) h_{9 / 2}\right)$, etc. In fact, each of these eigenstates form a pseudospin doublet. For instance, for specific value of $n=1,1 s_{1 / 2}$ with $\kappa=-1$ is considered as the partner of $0 d_{3 / 2}$ with $\kappa=2$. Thus, states that have pseudo orbital angular momentum $\tilde{l}$ quantum numbers, radial $n$ and $n-1$ with $j=\tilde{l}-1 / 2$ and $j=\widetilde{l}+1 / 2$, respectively, are degenerate [59].

\section{CONCLUSIONS}

We have studied the approximate bound state solutions of the Dirac equation for the GMP model with any arbitrary spin-orbit $\kappa$ state under the conditions of the spin (pseudospin) symmetry limitation by means of the NU method including a new improved approximation scheme to approximate the centrifugal (pseudo-centrifugal) barrier term. By setting $\Sigma(r)(\Delta(r))$ to the spherically symmetric GMP, we have derived the solutions of the Dirac equation for the relativistic energy eigenvalues and associated two-component spinor wave functions for arbitrary spin-orbit $\kappa$ state that provides an approximate solution to the spin- and pseudo-spin symmetry. The resulting solutions of the wave functions are being expressed in terms of the Jacobi polynomials (or hypergeometric functions). We have shown that the present spin symmetry can be easily reduced to the non-relativistic solution once we set $V(r)=S(r)$ (i.e., $\Delta(r)=0$ or $C_{s}=0$ ). The non-relativistic limits of our solution are obtained by imposing appropriate transformations and recalling $\kappa(\kappa+1) \rightarrow l(l+1)$ in the spin symmetry limits. Furthermore, when $\alpha \rightarrow 0$, our results can be reduced to the wellknown bound state solutions for the Kratzer potential model. If we choose the spin-orbit quantum number $\kappa=-1(\kappa=1)$ for spin (pseudospin) symmetry, the problem reduces to the exact $s(\widetilde{s})$-wave Dirac solution. We must point out that the numerical calculations for eigenenergy of the Dirac states involved in Eqs. (27) and (50) are sensitive to the choice of the parameters $C_{s}, C_{p s}, \alpha, r_{e}, D$ and $M$. It is found that the spin (pseudospin) limit Dirac eigenenergy valence (hole) states along with the two-component spinors are identical with the results obtained previously by other methods and works [15]. It is noticed that 
these results are obtained in a much simpler fashion than Ref. [15]. Finally, Eqs. (27) and (50) can be used to evaluate the binding energies of the GMP for diatomic molecules such as $\mathrm{CH}, \mathrm{CO}$ and $\mathrm{N}_{2}[2,56]$ in the relativistic framework with spin and pseudospin symmetry cases for any range of potential well $\alpha$. Equations. (41) and (57) can be also used to evaluate the binding energy of the Kratzer potential in the relativistic framework with spin and pseudospin symmetry cases at small range potential well $(\alpha \rightarrow 0)$.

\section{Acknowledgments}

We thank the anonymous referee and editors for their enlightening comments and suggestions which helped us to significantly improve the manuscript.

\section{Appendix A: Generalization of the NU method}

We briefly review the Nikiforov-Uvarov essential polynomials, root, eigenvalues and wave functions (see Eqs. (5) - (11) of Ref. [40]) being expressed in terms of the parameters $c_{i}$ $(i=1,2, \cdots, 13)$ together with $A, B$ and $C$ :

(i) The relevant constants:

$$
\begin{gathered}
c_{4}=\frac{1}{2}\left(1-c_{1}\right), c_{5}=\frac{1}{2}\left(c_{2}-2 c_{3}\right), c_{6}=c_{5}^{2}+A \\
c_{7}=2 c_{4} c_{5}-B, c_{8}=c_{4}^{2}+C, c_{9}=c_{3}\left(c_{7}+c_{3} c_{8}\right)+c_{6}, \\
c_{10}=c_{1}+2 c_{4}+2 \sqrt{c_{8}}-1>-1, c_{11}=1-c_{1}-2 c_{4}+\frac{2}{c_{3}} \sqrt{c_{9}}>-1, \\
c_{12}=c_{4}+\sqrt{c_{8}}>0, c_{13}=-c_{4}+\frac{1}{c_{3}}\left(\sqrt{c_{9}}-c_{5}\right)>0 .
\end{gathered}
$$

(ii) The essential polynomials:

$$
\begin{gathered}
\pi(z)=c_{4}+c_{5} z-\left[\left(\sqrt{c_{9}}+c_{3} \sqrt{c_{8}}\right) z-\sqrt{c_{8}}\right], \\
k=-\left(c_{7}+2 c_{3} c_{8}\right)-2 \sqrt{c_{8} c_{9}}, \\
\tau(z)=1-\left(c_{2}-2 c_{5}\right) z-2\left[\left(\sqrt{c_{9}}+c_{3} \sqrt{c_{8}}\right) z-\sqrt{c_{8}}\right], \\
\tau^{\prime}(z)=-2 c_{3}-2\left(\sqrt{c_{9}}+c_{3} \sqrt{c_{8}}\right)<0 .
\end{gathered}
$$


(iii) The energy equation:

$$
\left(c_{2}-c_{3}\right) n+c_{3} n^{2}-(2 n+1) c_{5}+(2 n+1)\left(\sqrt{c_{9}}+c_{3} \sqrt{c_{8}}\right)+c_{7}+2 c_{3} c_{8}+2 \sqrt{c_{8} c_{9}}=0 .
$$

(iv) The wave functions:

$$
\begin{gathered}
\rho(z)=z^{c_{10}}\left(1-c_{3} z\right)^{c_{11}}, \\
\phi(z)=z^{c_{12}}\left(1-c_{3} z\right)^{c_{13}}, c_{12}>0, c_{13}>0, \\
y_{n}(z)=P_{n}^{\left(c_{10}, c_{11}\right)}\left(1-2 c_{3} z\right), c_{10}>-1, c_{11}>-1, \\
F_{n \kappa}(z)=\mathcal{N}_{n \kappa} z^{c_{12}}\left(1-c_{3} z\right)^{c_{13}} P_{n}^{\left(c_{10}, c_{11}\right)}\left(1-2 c_{3} z\right),
\end{gathered}
$$

where $P_{n}^{(\mu, \nu)}(x), \mu>-1, \nu>-1$ and $x \in[-1,1]$ are the Jacobi polynomials with

$$
P_{n}^{(\alpha, \beta)}(1-2 s)=\frac{(\alpha+1)_{n}}{n !}{ }_{2} F_{1}(-n, 1+\alpha+\beta+n ; \alpha+1 ; s),
$$

and $\mathcal{N}_{n \kappa}$ is a normalization constants. Also, the above wave functions can be expressed in terms of the hypergeometric function as

$$
F_{n \kappa}(z)=\mathcal{N}_{n \kappa} z^{c_{12}}\left(1-c_{3} z\right)^{c_{13}}{ }_{2} F_{1}\left(-n, 1+c_{10}+c_{11}+n ; c_{10}+1 ; c_{3} z\right)
$$

where $c_{12}>0, c_{13}>0$ and $z \in\left[0,1 / c_{3}\right]$.

\section{Appendix B: Solving Quartic Energy Equation}

In order to solve the quartic equation (41b), the first step in the solution is to define the following variables

$$
u=a_{2}-\frac{3}{8} a_{3}^{2} ; v=a_{1}+\frac{1}{8} a_{3}^{3}-\frac{1}{2} a_{3} a_{2} ; w=a_{0}-\frac{3}{256} a_{3}^{4}+\frac{1}{16} a_{3}^{2} a_{2}-\frac{1}{4} a_{3} a_{1} ;
$$

which enable us to write down the related auxiliary cubic equation of the form:

$$
a \bar{E}_{n \kappa}^{3}+b \bar{E}_{n \kappa}^{2}+c \bar{E}_{n \kappa}+d=0
$$

with coefficients

$$
a=1, b=\frac{u}{2}, c=\frac{1}{16}\left(u^{2}-4 w\right), d=-\frac{v^{2}}{64} .
$$

The next step is solving the cubic equation (B2) by defining the variables

$$
f=\frac{c}{a}-\frac{1}{3} \frac{b^{2}}{a^{2}} ; g=\frac{2}{27} \frac{b^{3}}{a^{3}}-\frac{1}{3} \frac{b c}{a^{2}}+\frac{d}{a} ; h=\frac{1}{4} g^{2}+\frac{1}{27} f^{3},
$$


and then applying one of the following three cases:

(i) When all three roots are real $(h<0)$ : we define the variables

$$
s=\sqrt{\frac{1}{4} g^{2}-h} ; k=\cos ^{-1}\left(-\frac{g}{2 s}\right), F=\frac{1}{\sqrt[3]{s}}, H=\cos \left(\frac{k}{3}\right), G=\sqrt{3} \sin \left(\frac{k}{3}\right),
$$

where all the arguments in the trigonometric functions are in radians, to obtain the three possible roots of (B2) in the form

$$
\bar{E}_{n \kappa}^{(1)}=2 \frac{H}{F}-\frac{1}{3} \frac{b}{a} ; \bar{E}_{n \kappa}^{(2)}=F(H+G)+\frac{3 a}{b} ; \bar{E}_{n \kappa}^{(3)}=F(H-G)+\frac{3 a}{b},
$$

and hence the four energies of the original quartic equation (41b) take the forms

$$
\begin{aligned}
& E_{n \kappa}^{(1)}=\sqrt{\bar{E}_{n \kappa}^{(2)}}+\sqrt{\bar{E}_{n \kappa}^{(3)}}-\frac{g}{8 \sqrt{\bar{E}_{n \kappa}^{(2)} \bar{E}_{n \kappa}^{(3)}}}-\frac{1}{4} \frac{a_{3}}{a_{4}} ; E_{n \kappa}^{(2)}=\sqrt{\bar{E}_{n \kappa}^{(2)}}-\sqrt{\bar{E}_{n \kappa}^{(3)}}+\frac{g}{8 \sqrt{\bar{E}_{n \kappa}^{(2)} \bar{E}_{n \kappa}^{(3)}}}-\frac{1}{4} \frac{a_{3}}{a_{4}} ; \\
& E_{n \kappa}^{(3)}=-\sqrt{\bar{E}_{n \kappa}^{(2)}}+\sqrt{\bar{E}_{n \kappa}^{(3)}}+\frac{g}{8 \sqrt{\bar{E}_{n \kappa}^{(2)} \bar{E}_{n \kappa}^{(3)}}}-\frac{1}{4} \frac{a_{3}}{a_{4}} ; E_{n \kappa}^{(4)}=-\sqrt{\bar{E}_{n \kappa}^{(2)}}-\sqrt{\bar{E}_{n \kappa}^{(3)}}-\frac{g}{8 \sqrt{\bar{E}_{n \kappa}^{(2)} \bar{E}_{n \kappa}^{(3)}}}-\frac{1}{4} \frac{a_{3}}{a_{4}} .
\end{aligned}
$$

It is worth noting that whenever we have three real root we always choose the two non-zero roots; say, $\bar{E}_{n \kappa}^{(2)}$ and $\bar{E}_{n \kappa}^{(3)}$ in (B6) of the cubic equation.

(ii) When only one root is real $(h>0)$ : the definitions

$$
R=-\frac{1}{2} g+\sqrt{h} ; S=\sqrt[3]{R} ; T=-\frac{1}{2} g-\sqrt{h} ; U=\sqrt[3]{T}
$$

enable us to write down the three roots of (B2):

$$
\begin{gathered}
\bar{E}_{n \kappa}^{(1)}=S+U-\frac{b}{3 a} ; \bar{E}_{n \kappa}^{(2)}=-\frac{1}{2}(S+U)-\frac{b}{3 a}+i \frac{\sqrt{3}}{2}(S-U) ; \\
\bar{E}_{n \kappa}^{(3)}=-\frac{1}{2}(S+U)-\frac{b}{3 a}-i \frac{\sqrt{3}}{2}(S-U),
\end{gathered}
$$

and hence the four energies of Eq. (41b) are

$$
\begin{aligned}
& E_{n \kappa}^{(1)}=\sqrt{\bar{E}_{n \kappa}^{(2)}}+\sqrt{\bar{E}_{n \kappa}^{(3)}}-\frac{g}{8 \sqrt{\bar{E}_{n \kappa}^{(2)} \bar{E}_{n \kappa}^{(3)}}}-\frac{1}{4} \frac{a_{3}}{a_{4}} ; E_{n \kappa}^{(2)}=\sqrt{\bar{E}_{n \kappa}^{(2)}}-\sqrt{\bar{E}_{n \kappa}^{(3)}}+\frac{g}{8 \sqrt{\bar{E}_{n \kappa}^{(2)} \bar{E}_{n \kappa}^{(3)}}}-\frac{1}{4} \frac{a_{3}}{a_{4}} ; \\
& E_{n \kappa}^{(3)}=-\sqrt{\bar{E}_{n \kappa}^{(2)}}+\sqrt{\bar{E}_{n \kappa}^{(3)}}+\frac{g}{8 \sqrt{\bar{E}_{n \kappa}^{(2)} \bar{E}_{n \kappa}^{(3)}}}-\frac{1}{4} \frac{a_{3}}{a_{4}} ; E_{n \kappa}^{(4)}=-\sqrt{\bar{E}_{n \kappa}^{(2)}}-\sqrt{\bar{E}_{n \kappa}^{(3)}}-\frac{g}{8 \sqrt{\bar{E}_{n \kappa}^{(2)} \bar{E}_{n \kappa}^{(3)}}}-\frac{1}{4} \frac{a_{3}}{a_{4}} .
\end{aligned}
$$

It is worth noting that whenever we have one real root and two complex roots we always choose the two complex roots. 
(iii) When all three roots are real and equal $(f=g=h=0)$, then the roots of (B2):

$$
\bar{E}_{n \kappa}^{(1)}=\bar{E}_{n \kappa}^{(2)}=\bar{E}_{n \kappa}^{(3)}=-\sqrt[3]{\frac{d}{a}},
$$

and hence the four energies of the original quartic equation (41b) are

$$
E_{n \kappa}^{(1)}=-2 \sqrt[3]{\frac{d}{a}}-\frac{1}{4} \frac{a_{3}}{a_{4}} ; E_{n \kappa}^{(2)}=E_{n \kappa}^{(3)}=-\frac{1}{4} \frac{a_{3}}{a_{4}} ; E_{n \kappa}^{(4)}=2 \sqrt[3]{\frac{d}{a}}-\frac{1}{4} \frac{a_{3}}{a_{4}} .
$$

\section{Appendix C: Normalization constants}

The normalization constant, $\mathcal{N}_{n l}$ can be determined in closed form. We start by using the relation between the hypergeometric function and the Jacobi polynomials (see formula (8.962.1) in [49]):

$$
\begin{gathered}
{ }_{2} F_{1}\left(-n, n+\nu+\mu+1 ; \nu+1 ; \frac{1-x}{2}\right)=\frac{n !}{(\nu+1)_{n}} P_{n}^{(\nu, \mu)}(x), \\
(\nu+1)_{n}=\frac{\Gamma(n+\nu+1)}{\Gamma(\nu+1)}
\end{gathered}
$$

to rewrite the wave functions in $(32)$ as

$$
F_{n \kappa}(r)=\mathcal{N}_{n \kappa} \frac{n ! \Gamma\left(2 \varepsilon_{n \kappa}+1\right)}{\Gamma\left(n+2 \varepsilon_{n \kappa}+1\right)} e^{-\varepsilon_{n \kappa} \alpha r}\left(1-e^{-\alpha r}\right)^{\delta_{1}} P_{n}^{\left(2 \varepsilon_{n \kappa}, 2 \delta_{1}-1\right)}\left(1-2 e^{-\alpha r}\right) .
$$

From the normalization condition $\int_{0}^{\infty}\left[u_{n, l}(r)\right]^{2} d r=1$ and under the coordinate change $x=1-2 e^{-\alpha r}$, the normalization constant in (B2) is given by

$\mathcal{N}_{n \kappa}^{-2}=\frac{1}{\alpha}\left[\frac{n ! \Gamma\left(2 \varepsilon_{n \kappa}+1\right)}{\Gamma\left(n+2 \varepsilon_{n \kappa}+1\right)}\right]^{2} \int_{-1}^{1}\left(\frac{1-x}{2}\right)^{2 \varepsilon_{n \kappa}}\left(\frac{1+x}{2}\right)^{2 \delta_{1}-1}\left(\frac{1+x}{2}\right)\left[P_{n}^{\left(2 \varepsilon_{n \kappa}, 2 \delta_{1}-1\right)}(x)\right]^{2} d x$.

The calculation of this integral can be done by writting

$$
\frac{1+x}{2}=1-\left(\frac{1-x}{2}\right)
$$

and using the following two integrals (see formula (7.391.5) in [49]):

$$
\int_{-1}^{1}(1-x)^{\nu-1}(1+x)^{\mu}\left[P_{n}^{(\nu, \mu)}(x)\right]^{2} d x=2^{\nu+\mu} \frac{\Gamma(n+\nu+1) \Gamma(n+\mu+1)}{n ! \nu \Gamma(n+\nu+\mu+1)},
$$


which is valid for $\operatorname{Re}(\nu)>0$ and $\operatorname{Re}(\mu)>-1$ and (see formula (7.391.1) in [49]):

$$
\int_{-1}^{1}(1-x)^{\nu}(1+x)^{\mu}\left[P_{n}^{(\nu, \mu)}(x)\right]^{2} d x=2^{\nu+\mu+1} \frac{\Gamma(n+\nu+1) \Gamma(n+\mu+1)}{n ! \Gamma(n+\nu+\mu+1)(2 n+\nu+\mu+1)}
$$

which is valid for $\operatorname{Re}(\nu)>-1, \operatorname{Re}(\mu)>-1$. Thus, the normalization constant:

$$
\mathcal{N}_{n \kappa}=\frac{1}{\Gamma\left(2 \varepsilon_{n \kappa}+1\right)}\left[\frac{\alpha \varepsilon_{n \kappa}\left(n+\varepsilon_{n \kappa}+\delta_{1}\right)}{2\left(n+\delta_{1}\right)} \frac{\Gamma\left(n+2 \varepsilon_{n \kappa}+1\right) \Gamma\left(n+2 \varepsilon_{n \kappa}+2 \delta_{1}\right)}{n ! \Gamma\left(n+2 \delta_{1}\right)}\right]^{1 / 2},
$$

where $0 \leq n, \kappa<\infty$. In the $s$-wave $(\kappa=-1)$ case, the above result is written explicitly as

$$
\mathcal{N}_{n,-1}=\frac{1}{\Gamma(2 \eta+1)}\left[\frac{\alpha \eta(n+\eta+\delta)}{2(n+\delta)} \frac{\Gamma(n+2 \eta+1) \Gamma(n+2 \eta+2 \delta)}{n ! \Gamma(n+2 \delta)}\right]^{1 / 2} .
$$

Also, the non-relativistic normalization constant is therefore obtained as

$$
\mathcal{N}_{n l}=\frac{1}{\Gamma(2 \widetilde{\eta}+1)}\left[\frac{\alpha \widetilde{\eta}(n+\widetilde{\eta}+\widetilde{\delta})}{2(n+\widetilde{\delta})} \frac{\Gamma(n+2 \widetilde{\eta}+1) \Gamma(n+2 \widetilde{\eta}+2 \widetilde{\delta})}{n ! \Gamma(n+2 \widetilde{\delta})}\right]^{1 / 2}
$$


[1] Z.H. Deng and Y.P. Fan, Shandong Univ. J. 7, 162 (1957).

[2] L. Infeld and T.E. Hull, Rev. Mod. Phys. 23, 21 (1951); M.F. Manning and N. Rosen, Phys. Rev. 44, 953 (1933).

[3] J.W. Dabrowska, A. Khare and U.P. Sukhatma, J. Phys. A: Math. Gen. 21, L195 (1988); F. Cooper, A. Khare and U. Sukhatma, Phys. Rep. 251, 267 (1995); X. Zou, L.-Z. Yi and C.-S. Jia, Phys. Lett. A 346, 54 (2005).

[4] A.D. S. Mesa and C. Quesne and Y.F. Smirnov, J. Phys. A: Math. Gen. 31, 321 (1998).

[5] S. Flügge, P. Walger and A. Weiguny, J. Molec. Spectroscopy 23 (3), 243 (1967).

[6] C.S. Jia, Y. Li, Y. Sun, J.Y. Liu and L.T. Sun, Phys. Lett. A 311, 115 (2003).

[7] C.S. Jia, X.L. Zeng and L.T. Sun, Phys. Lett. A 294, 185 (2002).

[8] S. Codriansky, P. Cordero and S. Salamó, J. Phys. A: Math. Gen. 32, 6287 (1999).

[9] Z. Rong, H.G. Kjaergaard and M.L. Sage, Mol. Phys. 101, 2285 (2003).

[10] S.H. Dong and X.Y. Gu, J. Phys.: Conf. Ser. 96, 012109 (2008).

[11] R.L. Greene and C. Aldrich, Phys. Rev. A 14, 2363 (1976).

[12] Z. Rong and M.L. Sage, Interdiscip. Sci. Comput. Life Sci. 1, 163 (2009).

[13] S.L. Nyeo and I.C. Yang, Phys. Rev. E 63, 046109 (2001).

[14] C. Berkdemir, Nucl. Phys. A 770, 32 (2006); W.-C. Qiang, R.-S. Zhou and Y. Gao, J. Phys. A: Math. Theort. 40, 1677 (2007); A.D. Alhaidari, Phys. Rev. Lett. 89, 068901 (2002).

[15] L.H. Zhang, X.P. Li and C.S. Jia, Phys. Scr. 80, 035003 (2009).

[16] S.M. Ikhdair, C. Berkdemir and R. Sever, Spin and pseudospin symmetry along with orbital dependency of the Dirac-Hulthén problem, to appear in the Appl. Math. Comp. (2011).

[17] S.M. Ikhdair and R. Sever, Appl. Math. Comp. 216, 911 (2010).

[18] S.M. Ikhdair and R. Sever, Cent. Eur. J. Phys. 8 (4), 652 (2010); S.M. Ikhdair and R. Sever, Int. J. Mod. Phys. A 25 (20), 3941 (2010).

[19] C. Berkdemir, A. Berkdemir and R. Sever, J. Phys. A: Math. Gen. 39, 13455 (2006).

[20] A. Soylu, O. Bayrak and I. Boztosun, J. Phys. A: Math. Theor. 41, 065308 (2008); W.-C. Qiang, J.-Y. Wu and S.-H. Dong, Phys. Scr. 79, 065011 (2009); X.Y. Liu, G.F. Wei and C. Y. Long, Int. J. Theor. Phys. 48, 463 (2009).

[21] A. Arda, R. Sever, C. Tezcan and H. Akçay, Chin. Phys. Lett. 27, 040306 (2010); O. Bayrak 
and I. Boztosun, J. Phys. A: Math. Theort. 40, 11119 (2007).

[22] C.-S. Jia, P. Guo, Y.-F. Diao, L.-Z. Yi and X.-J. Xie, Eur. Phys. J. A 34, 41 (2007); Y. Xu, S. He and C.-S. Jia, J. Phys. A: Math. Theor. 41, 255302 (2008); H. Akcay, J. Phys. A: Math. Theor. 42, 198001 (2009).

[23] T. Chen, J.-Y. Liu and C.-S. Jia, Phys. Scr. 79, 055002 (2009).

[24] C.-S. Jia, J.-Y. Liu, L. He and L.-T. Sun, Phys. Scr. 75, 388 (2007).

[25] S.M. Ikhdair, J. Math. Phys. 51, 023525 (2010).

[26] O. Aydoğdu and R. Sever, Phys. Scr. 80, 015001 (2009).

[27] C. Berkdemir and R. Sever, J. Phys. A: Math. Theor. 41, 045302 (2008).

[28] C.S. Jia, J.Y. Liu, L. He and L.T. Sun, Phys. Scr. 75, 388 (2007).

[29] J.N. Ginocchio, Phys. Rev. C 69, 034318 (2004).

[30] J.N. Ginocchio, Phys. Rev. Lett. 78, 436 (1997).

[31] J.N. Ginocchio, Phys. Rep. 414, 165 (2005); J.N. Ginocchio, Phys. Rep. 315, 231 (1999).

[32] J.S. Bell and H. Ruegg, Nucl. Phys. B 98, 151 (1975).

[33] P.R. Page, T. Goldman and J.N. Ginocchio, Phys. Rev. Lett. 86, 204 (2001).

[34] A. Arima, M. Harvey and K. Shimizu, Phys. Lett. B 30, 517 (1969).

[35] K.T. Hecht and A. Adler, Nucl. Phys. A 137, 129 (1969).

[36] A.D. Alhaidari, H. Bahlouli and A. Al-Hasan, Phys. Lett. A 349, 87 (2006).

[37] J. Meng, K. Sugawara-Tanabe, S. Yamaji, P. Ring and A. Arima, Phys. Rev. C 58, R628 (1998).

[38] A.F. Nikiforov and V.B. Uvarov, Special Functions of Mathematical Physics, Birkhäuser: Basel, 1988.

[39] S.M. Ikhdair, Phys. Scr. 83, 015010 (2011).

[40] S.M. Ikhdair, Eur. Phys. J. A 39, 307 (2009); S.M. Ikhdair and R. Sever, Phys. Scr. 79, 035002 (2009).

[41] S.M. Ikhdair, Chem. Phys. 361, 9 (2009).

[42] W. Greiner, Relativistic Quantum Mechanics (Springer, Verlag, 1981).

[43] A.D. Alhaidari, H. Bahlouli and A. Al-Hasan, Phys. Lett. A 349, 87 (2006).

[44] C.-S. Jia, T. Chen, L.-G. Cui, Phys. Lett. A 373, 1621 (2009).

[45] Z.-Y. Chen, M. Li , C.-S. Jia, Mod. Phys. Lett. A 24 (23), 1863 (2009).

[46] Y.-F. Diao, L.-Z. Yi, T. Chen, C.-S. Jia, Mod. Phys. Lett. B 23, 2269 (2009). 
[47] T. Chen, J.-Y. Liu, C.-S. Jia, Phys. Scr. 79, 055002 (2009), T. Chen, Y.-F. Diao, C.-S. Jia, Phys. Scr. 79, 065014 (2009)..

[48] C.-S. Jia, Y.-F. Diao, L.-Z. Yi and T. Chen, Int. J. Mod. Phys. A 24 (24), 4519 (2009).

[49] M. Abramowitz and I.A. Stegun, Handbook of Mathematical Functions (Dover Publication, New York, 1972).

[50] I.S. Gradshtein and I.M. Ryzhik, Tables and integrals, series and products (New York, Academic, 1969).

[51] S.M. Ikhdair and R. Sever, J. Mol. Struct.: Theochem 855, 13 (2008).

[52] S.M. Ikhdair, Chin. J. Phys. 46, 291 (2008).

[53] S.M. Ikhdair, Eur. Phys. J. A 40, 143 (2009); S.M. Ikhdair and R. Sever, Appl. Math. Comp. 216,545 (2010).

[54] S.M. Ikhdair and R. Sever, J. Math. Chem. 45, 1137 (2009).

[55] C. Berkdemir and Y.-F. Cheng, Phys. Scr. 79, 035003 (2009).

[56] S.H. Dong and X.Y. Gu, J. Phys.: Conference Series 96, 012109 (2008).

[57] W. Lucha and F.F. Schöberl, Int. J. Mod. Phys. C 10, 607 (1999).

[58] S.M. Ikhdair and J. Abu-Hasna, Phys. Scr. 83, 025002 (2011).

[59] R. Lisboa, M. Malheiro, A.S. de Castro, P. Alberto and M. Fiolhais, Phys. Rev. C 69, 024319 (2004). 
FIG. 1: The GMP model with $D=15 \mathrm{fm}^{-1}$ for (a) various potential ranges $\alpha=0.05,0.15,0.30$ $\mathrm{fm}^{-1}$ along with $r_{e}=0.40 \mathrm{fm}$, and (b) various equilibrium inter-nuclear distances $r_{e}=0.40,0.80$, $1.20 \mathrm{fm}$ along with $\alpha=0.40 \mathrm{fm}^{-1}$.

FIG. 2: The upper and lower spinor wave functions, in the exact spin symmetry, for (a) $n=0$ $\left(0 p_{1 / 2}, 0 p_{3 / 2}\right)$ spin doublet eigenstates with $\kappa=1$ and $\kappa=-2$ and (b) $n=1\left(1 p_{1 / 2}, 1 p_{3 / 2}\right)$ spin doublet eigenstates with $\kappa=1$ and $\kappa=-2$.

TABLE I: Specific values of the constants in the solution of the GMP under spin symmetry.

\begin{tabular}{ll} 
Constant & Constant \\
\hline$c_{1}=1$ & $c_{2}=1$ \\
$c_{3}=1$ & $c_{4}=0$ \\
$c_{5}=-\frac{1}{2}$ & $c_{6}=\frac{1}{4}\left[1+4\left((2+b) \xi_{1}-\widetilde{E}_{n \kappa}\right)\right]$ \\
$c_{7}=2\left(\widetilde{E}_{n \kappa}-\xi_{1}\right)-\kappa(\kappa+1)$ & $c_{8}=-\widetilde{E}_{n \kappa}$ \\
$c_{9}=\frac{1}{4}\left[(1+2 \kappa)^{2}+4 b \xi_{1}\right]$ & $c_{10}=2 \eta_{1}=2 i \sqrt{\widetilde{E}_{n \kappa}}$ \\
$c_{11}=\sqrt{(1+2 \kappa)^{2}+4 b \xi_{1}}$ & $c_{12}=\eta_{1}$ \\
$c_{13}=\frac{1}{2}\left(1+\sqrt{(1+2 \kappa)^{2}+4 b \xi_{1}}\right)$ & $A=(2+b) \xi_{1}-\widetilde{E}_{n \kappa}$ \\
$B=-2\left(\widetilde{E}_{n \kappa}-\xi_{1}\right)-\kappa(\kappa+1)$ & $C=-\widetilde{E}_{n \kappa}$ \\
\hline
\end{tabular}


TABLE II: Specific values of the constants in the solution of the GMP under pseudospin symmetry.

\begin{tabular}{ll}
\hline Constant & Constant \\
\hline$c_{1}=1$ & $c_{2}=1$ \\
$c_{3}=1$ & $c_{4}=0$ \\
$c_{5}=-\frac{1}{2}$ & $c_{6}=\frac{1}{4}\left[1+4\left((2+b) \xi_{2}-\widetilde{E}_{n \kappa}\right)\right]$ \\
$c_{7}=2\left(\widetilde{E}_{n \kappa}-\xi_{2}\right)-\kappa(\kappa-1)$ & $c_{8}=\eta_{2}^{2}=-\widetilde{E}_{n \kappa}$ \\
$c_{9}=\frac{1}{4}\left[(1-2 \kappa)^{2}+4 b \xi_{2}\right]$ & $c_{10}=2 \eta_{2}=2 i \sqrt{\widetilde{E}_{n \kappa}}$ \\
$c_{11}=\sqrt{(1-2 \kappa)^{2}+4 b \xi_{2}}$ & $c_{12}=\eta_{2}$ \\
$c_{13}=\frac{1}{2}\left(1+\sqrt{(1-2 \kappa)^{2}+4 b \xi_{2}}\right)$ & $A=(2+b) \xi_{1}-\widetilde{E}_{n \kappa}$ \\
$B=-2\left(\widetilde{E}_{n \kappa}-\xi_{2}\right)-\kappa(\kappa-1)$ & $C=\eta_{2}^{2}=-\widetilde{E}_{n \kappa}$ \\
\hline
\end{tabular}


TABLE III: The Schrödinger bound state energy levels $E_{n l}$ (in au) of the GMP as functions of $\alpha$ and $r_{e}$ for various states with $D=15$ where $\hbar=\mu=1$.

$$
r_{e}=0.4 \quad r_{e}=0.8
$$

\begin{tabular}{llllllll} 
States $\alpha$ & Present & DG [53] LS [54] & Present & DG [53] LS [54] \\
\hline $2 p$ & 0.05 & 7.86080 & 7.8606 & 7.8628 & 4.14088 & 4.14068 & 4.14208
\end{tabular}

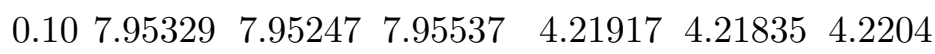

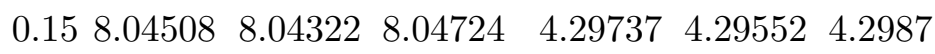

$\begin{array}{lllllll}0.20 & 8.13616 & 8.13287 & 8.13842 & 4.37551 & 4.37221 & 4.3769\end{array}$

$$
r_{e}=0.4
$$

$r_{e}=0.8$

$\begin{array}{lllllll}0.25 & 8.22656 & 8.22142 & 8.22892 & 4.45360 & 4.44845 & 4.4551\end{array}$

$\begin{array}{lllllll}0.30 & 8.31630 & 8.30889 & 8.31874 & 4.53166 & 4.52425 & 4.5332\end{array}$

$\begin{array}{llllllll}3 p & 0.05 & 10.9978 & 10.9976 & 10.9998 & 7.53279 & 7.53258 & 7.5350\end{array}$

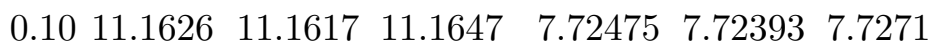

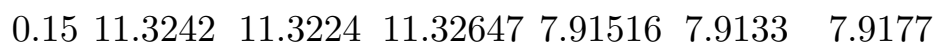

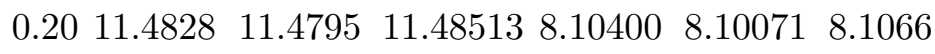

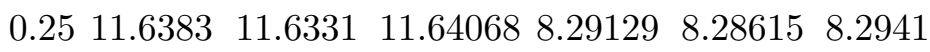

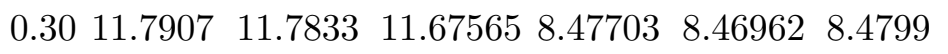

$\begin{array}{llllllll}3 d & 0.05 & 10.2160 & 10.2154 & 10.21651 & 5.73974 & 5.73913 & 5.7404\end{array}$

$\begin{array}{lllllll}0.10 & 10.3535 & 10.351 & 10.35409 & 5.84574 & 5.84327 & 5.8465\end{array}$

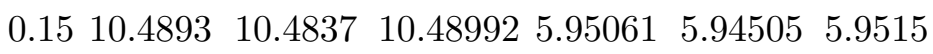

$\begin{array}{lllllll}0.20 & 10.6233 & 10.6135 & 10.62403 & 6.05441 & 6.04453 & 6.0553\end{array}$

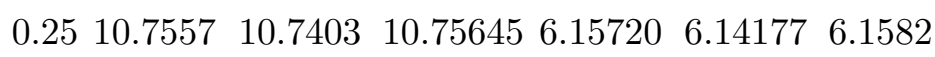

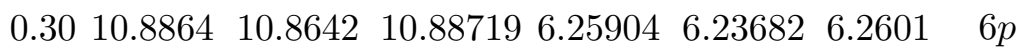

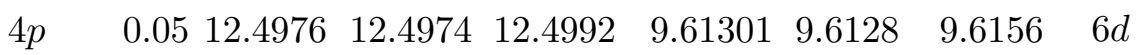

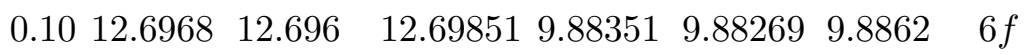

$\begin{array}{llllllll}0.15 & 12.8883 & 12.8865 & 12.8901 & 10.1485 & 10.1467 & 10.1514 & 6 g\end{array}$

$\begin{array}{lllllll}0.20 & 13.0722 & 13.0689 & 13.0740 & 10.4080 & 10.4047 & 10.4111\end{array}$

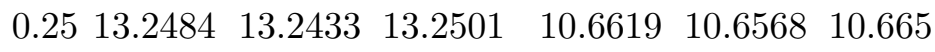

$\begin{array}{llllllll}4 d & 0.05 & 12.0983 & 12.0977 & 12.0989 & 8.49334 & 8.49272 & 8.4948\end{array}$

$\begin{array}{lllllll}0.10 & 12.2850 & 12.2825 & 12.2857 & 8.70708 & 8.70461 & 8.7087\end{array}$

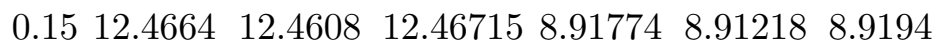

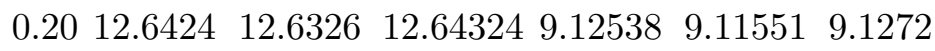

States $\alpha$ Present DG [53] LS [54] Present

$\begin{array}{llllll}\text { uf } & 0.05 & 11.8208 & 11.8195 & 11.8209 & 7.43469\end{array}$

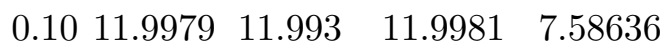

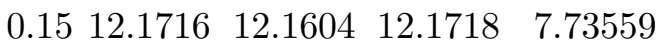

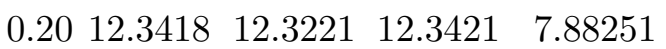

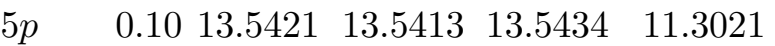

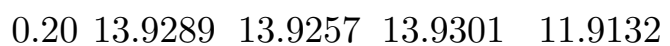

$0.1013 .3068 \quad 13.3043 \quad 13.3075 \quad 10.5201$

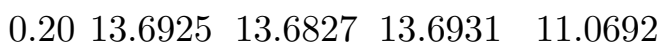

$\begin{array}{lllll}0.10 & 13.1475 & 13.1426 & 13.1478 & 9.7966\end{array}$

$\begin{array}{lllll}0.20 & 13.5332 & 13.5134 & 13.5333 & 10.2728\end{array}$

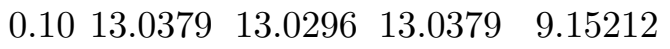

$0.2013 .4267 \quad 13.3938 \quad 13.426679 .55246$

$\begin{array}{lllll}0.10 & 14.0521 & 14.0513 & 14.0530 & 12.2798\end{array}$

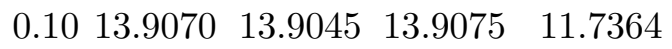

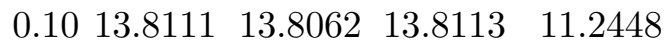

$\begin{array}{lllll}0.10 & 13.7465 & 13.7383 & 13.7466 & 10.8152\end{array}$ 
TABLE IV: The Schrödinger bound state energy levels $E_{n l}$ (in au) of the Kratzer potential as a function of $r_{e}$ for various states with $D=15$ where $\hbar=\mu=1$.

\begin{tabular}{llllll}
\hline State $/ r_{e}=$ & 0.1 & 0.4 & 0.8 & 1.0 & 1.5 \\
\hline $2 p$ & 13.9765 & 7.76759 & 4.06249 & 3.21339 & 2.07749 \\
$3 p$ & 14.5308 & 10.8298 & 7.33925 & 6.26836 & 4.56776 \\
$3 d$ & 14.5192 & 10.0766 & 5.63256 & 4.41694 & 2.74032 \\
$4 p$ & 14.7319 & 12.2908 & 9.33707 & 8.27299 & 6.40188 \\
$4 d$ & 14.7269 & 11.9062 & 8.27643 & 7.04418 & 5.05045 \\
$4 f$ & 14.7246 & 11.6401 & 7.28048 & 5.81633 & 3.61468 \\
$5 p$ & 14.8268 & 13.0996 & 10.6443 & 9.65890 & 7.79162 \\
$5 d$ & 14.8242 & 12.8774 & 9.94062 & 8.80199 & 6.76420 \\
$5 f$ & 14.8230 & 12.7278 & 9.29975 & 7.96875 & 5.69315 \\
$5 g$ & 14.8224 & 12.6242 & 8.73653 & 7.18597 & 4.60125 \\
$6 p$ & 14.8789 & 13.5937 & 11.5460 & 10.6568 & 8.86976 \\
$6 d$ & 14.8774 & 13.4539 & 11.0555 & 10.0356 & 8.07050 \\
$6 f$ & 14.8768 & 13.3616 & 10.6191 & 9.44444 & 7.25037 \\
$6 g$ & 14.8764 & 13.2985 & 10.2430 & 8.89964 & 6.42694 \\
\hline
\end{tabular}


TABLE V: The eigeenergies of the Dirac valence states in units of $\mathrm{fm}^{-1}$ for several values of $n$ and $\kappa$ with the parameters $M=1.0 \mathrm{fm}^{-1}$ and $D=15 \mathrm{fm}^{-1}$ in the case of exact spin symmetry limit $\left(C_{s}=0 \mathrm{fm}^{-1}\right)$. We have set $\hbar=c=1$.

\begin{tabular}{|c|c|c|c|c|c|c|}
\hline$l$ & $n, \kappa<0$ & $n L_{j=l+1 / 2}$ & $E_{n, \kappa<0}$ & \multicolumn{2}{|c|}{$n, \kappa>0 n L_{j=l-1 / 2}$} & $E_{n, \kappa>0}$ \\
\hline \multicolumn{7}{|c|}{$\alpha=0.10 \mathrm{fm}^{-1}, r_{e}=0.40 \mathrm{fm}$} \\
\hline 1 & $0,-2$ & $0 p_{3 / 2}$ & 5.5791076 & 0,1 & $0 p_{1 / 2}$ & 5.5791076 \\
\hline 2 & $0,-3$ & $0 d_{5 / 2}$ & 6.8118605 & 0,2 & $0 d_{3 / 2}$ & 6.8118605 \\
\hline 3 & $0,-4$ & $0 f_{7 / 2}$ & 8.0171073 & 0,3 & $0 f_{5 / 2}$ & 8.0171073 \\
\hline 4 & $0,-5$ & $0 g_{9 / 2}$ & 9.1025175 & 0,4 & $0 g_{7 / 2}$ & 9.1025175 \\
\hline 1 & $1,-2$ & $1 p_{3 / 2}$ & 8.1823677 & 1,1 & $1 p_{1 / 2}$ & 8.1823677 \\
\hline 2 & $1,-3$ & $1 d_{5 / 2}$ & 8.8815340 & 1,2 & $1 d_{3 / 2}$ & 8.8815340 \\
\hline 3 & $1,-4$ & $1 f_{7 / 2}$ & 9.6603105 & 1,3 & $1 f_{5 / 2}$ & 9.6603105 \\
\hline 4 & $1,-5$ & $1 g_{9 / 2}$ & 10.4200196 & 1,4 & $1 g_{7 / 2}$ & 10.4200196 \\
\hline \multicolumn{7}{|c|}{$\alpha=0.30 \mathrm{fm}^{-1}, r_{e}=0.40 \mathrm{fm}$} \\
\hline 1 & $0,-2$ & $0 p_{3 / 2}$ & 5.7078594 & 0,1 & $0 p_{1 / 2}$ & 5.7078594 \\
\hline 2 & $0,-3$ & $0 d_{5 / 2}$ & 6.9646771 & 0,2 & $0 d_{3 / 2}$ & 6.9646771 \\
\hline 3 & $0,-4$ & $0 f_{7 / 2}$ & 8.2121326 & 0,3 & $0 f_{5 / 2}$ & 8.2121326 \\
\hline 4 & $0,-5$ & $0 g_{9 / 2}$ & 9.3506414 & 0,4 & $0 g_{7 / 2}$ & 9.3506414 \\
\hline 1 & $1,-2$ & $1 p_{3 / 2}$ & 8.4626850 & 1,1 & $1 p_{1 / 2}$ & 8.4626850 \\
\hline 2 & $1,-3$ & $1 d_{5 / 2}$ & 9.1762544 & 1,2 & $1 d_{3 / 2}$ & 9.1762544 \\
\hline 3 & $1,-4$ & $1 f_{7 / 2}$ & 9.9831712 & 1,3 & $1 f_{5 / 2}$ & 9.9831712 \\
\hline 4 & $1,-5$ & $1 g_{9 / 2}$ & 10.7812870 & 1,4 & $1 g_{7 / 2}$ & 10.7812870 \\
\hline \multicolumn{7}{|c|}{$\alpha=0.10 \mathrm{fm}^{-1}, r_{e}=0.80 \mathrm{fm}$} \\
\hline 1 & $0,-2$ & $0 p_{3 / 2}$ & 3.6831690 & 0,1 & $0 p_{1 / 2}$ & 3.6831690 \\
\hline 2 & $0,-3$ & $0 d_{5 / 2}$ & 4.3378367 & 0,2 & $0 d_{3 / 2}$ & 4.3378367 \\
\hline 3 & $0,-4$ & $0 f_{7 / 2}$ & 5.0775317 & 0,3 & $0 f_{5 / 2}$ & 5.0775317 \\
\hline 4 & $0,-5$ & $0 g_{9 / 2}$ & 5.8291637 & 0,4 & $0 g_{7 / 2}$ & 5.8291637 \\
\hline 1 & $1,-2$ & $1 p_{3 / 2}$ & 5.8388616 & 1,1 & $1 p_{1 / 2}$ & 5.8388616 \\
\hline 2 & $1,-3$ & $1 d_{5 / 2}$ & 6.2180443 & 1,2 & $1 d_{3 / 2}$ & 6.2180443 \\
\hline 3 & $1,-4$ & $1 f_{7 / 2}$ & 6.6999906 & 1,3 & $1 f_{5 / 2}$ & 6.6999906 \\
\hline 4 & $1,-5$ & $1 g_{9 / 2}$ & 7.2334098 & 1,4 & $1 g_{7 / 2}$ & 7.2334098 \\
\hline
\end{tabular}


TABLE VI: The eigenenergies of the Dirac hole states in units of $\mathrm{fm}^{-1}$ for several values of $n$ and $\kappa$ with the parameters $M=1.0 \mathrm{fm}^{-1}$ and $D=15 \mathrm{fm}^{-1}$ in the case of the pseudospin limit. We have set $\hbar=c=1$.

\begin{tabular}{|c|c|c|c|c|c|c|c|c|}
\hline$\tilde{l}$ & $n, \kappa<0$ & $(l, j)$ & $E_{n, \kappa<0}$ & $E_{n, \kappa<0}$ & \multicolumn{2}{|c|}{$n-1, \kappa>0(l+2, j+1)$} & \multirow{2}{*}{$\begin{array}{l}E_{n-1, \kappa>0} \\
C_{p s}=0\end{array}$} & \multirow{2}{*}{$\begin{array}{l}E_{n-1, \kappa>0} \\
C_{p s}=5.0\end{array}$} \\
\hline & $r_{e}=0.40 \mathrm{fm}$ & & $C_{p s}=0$ & $C_{p s}=5.0$ & & & & \\
\hline 1 & $1,-1$ & $1 s_{1 / 2}$ & 7.1975980 & 9.0681299 & 0,2 & $0 d_{3 / 2}$ & 7.1975980 & 9.0681299 \\
\hline 2 & $1,-2$ & $1 p_{3 / 2}$ & 7.9184956 & 9.7118773 & 0,3 & $0 f_{5 / 2}$ & 7.9184956 & 9.7118773 \\
\hline 3 & $1,-3$ & $1 d_{5 / 2}$ & 8.6773995 & 10.3429382 & 0,4 & $0 g_{7 / 2}$ & 8.6773995 & 10.3429382 \\
\hline 4 & $1,-4$ & $1 f_{7 / 2}$ & 9.3887565 & 10.9088876 & 0,5 & $0 h_{9 / 2}$ & 9.3887565 & 10.9088876 \\
\hline 1 & $2,-1$ & $2 s_{1 / 2}$ & 8.7054710 & 10.1707692 & 1,2 & $1 d_{3 / 2}$ & 8.7054710 & 10.1707692 \\
\hline 2 & $2,-2$ & $2 p_{3 / 2}$ & 9.1768973 & 10.6114449 & 1,3 & $1 f_{5 / 2}$ & 9.1768973 & 10.6114449 \\
\hline 3 & $2,-3$ & $2 d_{5 / 2}$ & 9.7101038 & 11.0728353 & 1,4 & $1 g_{7 / 2}$ & 9.7101038 & 11.0728353 \\
\hline 4 & $2,-4$ & $2 f_{7 / 2}$ & 10.2352574 & 11.5024429 & 1,5 & $1 h_{9 / 2}$ & 10.2352574 & 11.5024429 \\
\hline \multicolumn{2}{|c|}{$\alpha=0.30 \mathrm{fm}^{-1}, r_{e}=0.40 \mathrm{fm}$} & & $C_{p s}=0$ & \multicolumn{2}{|l|}{$C_{p s}=-5.0$} & & $C_{p s}=0$ & $C_{p s}=-5.0$ \\
\hline 1 & $1,-1$ & $1 s_{1 / 2}$ & 7.4924218 & 6.2513272 & 0,2 & $0 d_{3 / 2}$ & 7.4924218 & 6.2513272 \\
\hline 2 & $1,-2$ & $1 p_{3 / 2}$ & 8.2327097 & 6.9536060 & 0,3 & $0 f_{5 / 2}$ & 8.2327097 & 6.9536060 \\
\hline 3 & $1,-3$ & $1 d_{5 / 2}$ & 9.0263198 & 7.7574142 & 0,4 & $0 g_{7 / 2}$ & 9.0263198 & 7.7574142 \\
\hline 4 & $1,-4$ & $1 f_{7 / 2}$ & 9.7816289 & 8.5599024 & 0,5 & $0 h_{9 / 2}$ & 9.7816289 & 8.5599024 \\
\hline 1 & $2,-1$ & $2 s_{1 / 2}$ & 9.1118917 & 8.0615654 & 1,2 & $1 d_{3 / 2}$ & 9.1118917 & 8.0615654 \\
\hline 2 & $2,-2$ & $2 p_{3 / 2}$ & 9.5965089 & 8.5254559 & 1,3 & $1 f_{5 / 2}$ & 9.5965089 & 8.5254559 \\
\hline 3 & $2,-3$ & $2 d_{5 / 2}$ & 10.1539358 & 9.0879925 & 1,4 & $1 g_{7 / 2}$ & 10.1539358 & 9.0879925 \\
\hline 4 & $2,-4$ & $2 f_{7 / 2}$ & 10.7110039 & 9.6762585 & 1,5 & $1 h_{9 / 2}$ & 10.7110039 & 9.6762585 \\
\hline \multicolumn{3}{|c|}{$\alpha=0.10 \mathrm{fm}^{-1}, r_{e}=0.80 \mathrm{fm}$} & $C_{p s}=0$ & \multicolumn{2}{|l|}{$C_{p s}=-10.0$} & & $C_{p s}=0$ & $C_{p s}=-10.0$ \\
\hline 1 & $1,-1$ & $1 s_{1 / 2}$ & 4.9972498 & 2.8369925 & 0,2 & $0 d_{3 / 2}$ & 4.9972498 & 2.8369925 \\
\hline 2 & $1,-2$ & $1 p_{3 / 2}$ & 5.4281366 & 3.1294486 & 0,3 & $0 f_{5 / 2}$ & 5.4281366 & 3.1294486 \\
\hline 3 & $1,-3$ & $1 d_{5 / 2}$ & 5.9435202 & 3.5245459 & 0,4 & $0 g_{7 / 2}$ & 5.9435202 & 3.5245459 \\
\hline 4 & $1,-4$ & $1 f_{7 / 2}$ & 6.4876355 & 3.9879463 & 0,5 & $0 h_{9 / 2}$ & 6.4876355 & 3.9879463 \\
\hline 1 & $2,-1$ & $2 s_{1 / 2}$ & 6.3778620 & 4.3337279 & 1,2 & $1 d_{3 / 2}$ & 6.3778620 & 4.3337279 \\
\hline 2 & $2,-2$ & $2 p_{3 / 2}$ & 6.6705985 & 4.5479115 & 1,3 & $1 f_{5 / 2}$ & 6.6705985 & 4.5479115 \\
\hline 3 & $2,-3$ & $2 d_{5 / 2}$ & 7.0449439 & 4.8443318 & 1,4 & $1 g_{7 / 2}$ & 7.0449439 & 4.8443318 \\
\hline 4 & $2,-4$ & $2 f_{7 / 2}$ & 7.4613765 & 5.2011464 & 1,5 & $1 h_{9 / 2}$ & 7.4613765 & 5.2011464 \\
\hline
\end{tabular}

